\title{
Positive radial solutions of $n$-dimensional elliptic systems with indefinite weight functions and $n$ parameters
}

Meiqiang Feng ${ }^{1 *}$ (D) and Ping Li ${ }^{1}$

"Correspondence:
meiqiangfeng@sina.com
'School of Applied Science, Beijing
Information Science \& Technology
University, Beijing, People's Republic
of China
of China

\section{Abstract}

Under simple conditions on $\mathbf{f}$ and $\mathbf{a}$, we show the existence of positive radial solutions for the $n$-dimensional elliptic differential system

$$
\left\{\begin{array}{l}
\Delta \mathbf{u}(\mathbf{x})+\boldsymbol{\Lambda} \mathbf{a}(|\mathbf{x}|) \mathbf{f}(\mathbf{u}(\mathbf{x}))=\mathbf{0}, \quad R_{1}<|\mathbf{x}|<R_{2} \\
\left.\mathbf{u}\right|_{|\mathbf{x}|=R_{1}}=\left.\mathbf{u}\right|_{|\mathbf{x}|=R_{2}}=\mathbf{0} .
\end{array}\right.
$$

Here $\Delta$ denotes the Laplace operator, $\mathbf{f}(\mathbf{x})=\left(f_{1}(\mathbf{x}), \ldots, f_{i}(\mathbf{x}), \ldots, f_{n}(\mathbf{x})\right)^{T}$, $\mathbf{x}=\left(x_{1}, x_{2}, \ldots, x_{n}\right)^{T}, \mathbf{u}(\mathbf{x})=\left(u_{1}(\mathbf{x}), \ldots, u_{i}(\mathbf{x}), \ldots, u_{n}(\mathbf{x})\right)^{T}, \Delta \mathbf{u}(\mathbf{x})=$ $\left(\Delta u_{1}(\mathbf{x}), \ldots, \Delta u_{i}(\mathbf{x}), \ldots, \Delta u_{n}(\mathbf{x})\right)^{T}, \boldsymbol{\Lambda}=\operatorname{diag}\left[\lambda_{1}, \ldots, \lambda_{i}, \ldots, \lambda_{n}\right], \mathbf{a}(|\mathbf{x}|)=$ $\operatorname{diag}\left[a_{1}(|\mathbf{x}|), \ldots, a_{i}(|\mathbf{x}|), \ldots, a_{n}(|\mathbf{x}|)\right], R_{2}>R_{1}>0, \mathbf{x} \in \mathcal{R}^{n}, n \geq 2$. The interest is that $\mathbf{a}(|\mathbf{x}|)$ is allowed to change sign on $\left[R_{1}, R_{2}\right]$, which needs some new ingredients in the arguments. An example is also given to illustrate the main results.

Keywords: Positive radial solutions; $n$-dimensional elliptic systems; Indefinite weight function; $n$ parameters

\section{Introduction}

In this paper, we analyze the existence and multiplicity of positive radial solutions for the following $n$-dimensional elliptic differential system:

$$
\left\{\begin{array}{l}
\Delta \mathbf{u}(\mathbf{x})+\boldsymbol{\Lambda} \mathbf{a}(|\mathbf{x}|) f(\mathbf{u}(\mathbf{x}))=\mathbf{0}, \quad R_{1}<|\mathbf{x}|<R_{2}, \\
\left.\mathbf{u}\right|_{|\mathbf{x}|=R_{1}}=\left.\mathbf{u}\right|_{|\mathbf{x}|=R_{2}}=\mathbf{0},
\end{array}\right.
$$

where $\Delta$ denotes the Laplace operator, $R_{2}>R_{1}>0, \mathbf{x} \in \mathcal{R}^{n}, n \geq 2$, $\mathbf{a}(|\mathbf{x}|)$ is allowed to change sign on $\left[R_{1}, R_{2}\right]$, and

$$
\begin{aligned}
& \mathbf{x}=\left(x_{1}, x_{2}, \ldots, x_{n}\right)^{T}, \\
& \mathbf{u}(\mathbf{x})=\left(u_{1}(\mathbf{x}), \ldots, u_{i}(\mathbf{x}), \ldots, u_{n}(\mathbf{x})\right)^{T}, \\
& \Delta \mathbf{u}(\mathbf{x})=\left(\Delta u_{1}(\mathbf{x}), \ldots, \Delta u_{i}(\mathbf{x}), \ldots, \Delta u_{n}(\mathbf{x})\right)^{T}, \\
& \boldsymbol{\Lambda}=\operatorname{diag}\left[\lambda_{1}, \ldots, \lambda_{i}, \ldots, \lambda_{n}\right],
\end{aligned}
$$




$$
\begin{aligned}
& \mathbf{a}(|\mathbf{x}|)=\operatorname{diag}\left[a_{1}(|\mathbf{x}|), \ldots, a_{i}(|\mathbf{x}|), \ldots, a_{n}(|\mathbf{x}|)\right] \\
& \mathbf{f}(\mathbf{x})=\left(f_{1}(\mathbf{x}), \ldots, f_{i}(\mathbf{x}), \ldots, f_{n}(\mathbf{x})\right)^{T}
\end{aligned}
$$

where we understand $f_{i}(\mathbf{x})$ to mean $f_{i}\left(x_{1}, x_{2}, \ldots, x_{n}\right), i=1,2, \ldots, n$.

Therefore, system (1.1) means that $(i=1,2, \ldots, n)$

$$
\left\{\begin{array}{l}
\Delta u_{i}(\mathbf{x})+\lambda_{i} a_{i}(|\mathbf{x}|) f_{i}(\mathbf{u}(\mathbf{x}))=0, \quad R_{1}<|\mathbf{x}|<R_{2} \\
\left.u_{i}\right|_{|\mathbf{x}|=R_{1}}=\left.u_{i}\right|_{|\mathbf{x}|=R_{2}}=0
\end{array}\right.
$$

Let $J=[0,1], \mathcal{R}_{+}=[0,+\infty), \mathcal{R}=(-\infty,+\infty), \mathcal{R}_{+}^{n}=\underbrace{\mathcal{R}_{+} \times \mathcal{R}_{+} \times \cdots \times \mathcal{R}_{+}}_{n}$. By a positive radial solution $\mathbf{u}^{*}$ of system (1.1) we understand a solution $\mathbf{u}^{*}$ with $u_{i}^{*} \geq 0(i=1,2, \ldots, n)$ and either $u_{i}^{*} \not \equiv 0(i=1,2, \ldots, n)$. By the maximum principle, each nontrivial component of $\mathbf{u}^{*}$ is thus positive in $\Omega=\left\{\mathbf{x} \in \mathcal{R}^{n}: R_{1}<|\mathbf{x}|<R_{2}, R_{1}, R_{2}>0\right\}$. For $\mathbf{x}, \mathbf{y} \in \mathcal{R}^{n}$, we define $\mathbf{x} \leq \mathbf{y}$ if and only if $x_{i} \leq y_{i}, i=1,2, \ldots, n$.

The study of boundary value problems with positive solutions has attracted recently the attention of different researchers and it is a topic of current interest; see [1-16], and the references therein.

At the same time, we note that the existence and multiplicity of solutions to the elliptic differential systems:

$$
\left\{\begin{array}{l}
\Delta u+k_{1}(|x|) f(u, v)=0, \\
\Delta v+k_{2}(|x|) g(u, v)=0,
\end{array}\right.
$$

under different boundary conditions have been studied extensively in the past decades (see [17-36]). Kawano and Kusano [17] gave sufficient conditions which guarantee the existence of entire solutions of (1.3) by means of the method of sub- and super-solutions. By applying the linking theorem and with the assistance of the Nehari manifold, Benrhouma [18] showed the existence of at least two solutions of (1.3) in the whole space $\mathcal{R}^{n}$. Serrin and Zou [37] gave an excellent survey on the existence results for problem (1.3).

However, there is almost no paper dealing with the $n$-dimensional elliptic differential system with indefinite weight functions and parameters; for instance, see [38-41] and the references therein. Dalmasso [38] investigated the existence and uniqueness of positive solutions for the following elliptic system:

$$
\begin{cases}\Delta u+g(v)=0 & \text { in } \Omega, \\ \Delta v+f(u)=0 & \text { in } \Omega, \\ u=v=0 & \text { on } \Omega,\end{cases}
$$

where $\Omega \subset \mathcal{R}^{n}(n \geq 1)$ denotes a bounded domain of class $C^{2, \alpha}, \alpha \in(0,1]$. Precup [39] considered the existence, localization and multiplicity of positive radial solutions of the elliptic differential system:

$$
\left\{\begin{array}{l}
\Delta u_{1}+f_{1}(|x|) g_{1}\left(u_{1}, u_{2}\right)=0, \\
\Delta u_{2}+f_{2}(|x|) g_{2}\left(u_{1}, u_{2}\right)=0,
\end{array}\right.
$$


in $\Omega:=\left\{x \in \mathcal{R}^{n}:|x|>r_{0}\right\}(n \geq 3)$, under the conditions

$$
u_{1}=u_{2}=0 \quad \text { for }|x|=r_{0} \quad \text { and } \quad u_{1}, u_{2} \rightarrow 0 \quad \text { as }|x| \rightarrow \infty
$$

Recently, in [40], Maniwa studied the uniqueness and existence of positive solutions for the following elliptic differential system:

$$
\begin{cases}-\Delta u_{i}=\prod_{j=1}^{N} u_{j}^{p_{i j}} & \text { in } \Omega \\ u_{i}=0 & \text { on } \Omega, i=1,2, \ldots, N\end{cases}
$$

where $p_{i j}(1 \leq i, j \leq N)$ are nonnegative constants and $\Omega \subset \mathcal{R}^{n}(n \geq 1)$ denotes a bounded domain of class $C^{2, \alpha}, \alpha \in(0,1)$.

To the best of our knowledge, in the literature there are no articles on multiple radial positive solutions for the analogous of $n$-dimensional elliptic differential system with indefinite weights and $n$ parameters. More precisely, the study of $\boldsymbol{\Lambda} \neq 1$, and a changing sign on $\left[R_{1}, R_{2}\right]$ is still open for the elliptic systems. Specially, comparing with $[17-39,41,42]$, the main features of this paper are as follows.

(i) $\lambda_{i}>0$ is available, not only $\lambda_{i} \equiv 1, i=1,2, \ldots, n$.

(ii) $\mathbf{a}(|\mathbf{x}|)$ being allowed to change sign on $\left[R_{1}, R_{2}\right]$ is considered.

(iii) $n$-dimensional elliptic system is investigated.

In [40], the author considered $n$-dimensional elliptic system and obtained several excellent results of uniqueness and existence of positive solutions, but Maniwa only studied the case $\lambda_{i} \equiv 1$ and $a_{i}(|\mathbf{x}|) \equiv 1(i=1,2, \ldots, n)$. On the other hand, in [43], Yao pointed out that it is of particular mathematical interest when the weight function $\mathbf{a}(|\mathbf{x}|)$ is allowed to change sign on $\left[R_{1}, R_{2}\right]$. Therefore, the present work is new and significant.

In this paper, we always suppose that the following conditions hold:

1. $f_{i}: \mathcal{R}_{+}^{n} \rightarrow \mathcal{R}_{+}$is continuous and there exists $0<c_{i} \leq 1$ such that

$$
f_{i}(\mathbf{x}) \geq c_{i} \varphi_{i}(\mathbf{x}), \quad \mathbf{x} \in \mathcal{R}_{+}^{n},
$$

where $\varphi_{i}(\mathbf{x})=\max \left\{f_{i}(\mathbf{y}): 0 \leq \mathbf{y} \leq \mathbf{x}\right\}, i=1,2, \ldots, n$.

2. $a_{i}:\left[R_{1}, R_{2}\right] \rightarrow \mathcal{R}$ is continuous and there exists $R_{1}<\zeta<R_{2}$ such that

$$
a_{i}(r) \geq 0, \quad r \in\left[R_{1}, \zeta\right] ; \quad a_{i}(r) \leq 0, \quad r \in\left[\zeta, R_{2}\right], i=1,2, \ldots, n
$$

Moreover, $a_{i}(r)(i=1,2, \ldots, n)$ does not vanish identically on any subintervals of $\left[R_{1}, R_{2}\right]$.

In Sect. 2 we list several preliminary results that will be used in the subsequent sections. Section 3 is devoted to stating and proving the main results. Several special cases and an example are also given in Sect. 4.

\section{Preliminaries}

Looking for radial solutions, let us first introduce the radial coordinates form of system (1.2); for details to see Precup [39] and Lee [44]. By the radial variable $r=|x|$, we can write 
(1.2) as

$$
\left\{\begin{array}{l}
u_{i}^{\prime \prime}(r)+\frac{n-1}{r} u_{i}^{\prime}(r)+\lambda_{i} a_{i}(r) f_{i}(\mathbf{u}(r))=0, \quad R_{1}<r<R_{2} \\
u_{i}\left(R_{1}\right)=u_{i}\left(R_{2}\right)=0
\end{array}\right.
$$

where $i=1,2, \ldots, n$.

Let

$$
s(r)=-\int_{r}^{R_{2}} \frac{d t}{t^{n-1}} .
$$

Then

$$
s\left(R_{1}\right)=-\int_{R_{1}}^{R_{2}} \frac{d t}{t^{n-1}}=: m
$$

and $s\left(R_{2}\right)=0$.

Set $v_{i}(s)=u_{i}(r(s))$. Then taking the derivative of this equation with respect to $r$, and together with (2.2), we get

$$
\begin{aligned}
& u_{i r}^{\prime}=v_{i s}^{\prime} \times s^{\prime}(r)=v_{i s}^{\prime} \times \frac{1}{r^{n-1}}, \\
& u_{i r r}^{\prime \prime}=v_{i s s}^{\prime \prime} \times \frac{1}{r^{2 n-2}}+v_{i s}^{\prime} \times(1-n) r^{2-n} .
\end{aligned}
$$

For convenience, we write $v_{i s s}^{\prime \prime}$ as $v_{i}^{\prime \prime}(s)$. Thus submitting $u_{i r r}^{\prime}$ and $u_{i r}^{\prime \prime}$ into (2.1), we get

$$
\left\{\begin{array}{l}
v_{i}^{\prime \prime}(s)+\lambda_{i} r^{2(n-1)}(s) a_{i}(r(s)) f_{i}(\mathbf{v}(s))=0, \quad m<s<0, \\
v_{i}(m)=v_{i}(0)=0
\end{array}\right.
$$

where $i=1,2, \ldots, n, \mathbf{v}=\left(v_{1}, v_{2}, \ldots, v_{n}\right)^{T}$.

Let $t=\frac{m-s}{m}$. Then $s=m(1-t)$. Set $w_{i}(t)=v_{i}(s)$. Then similarly system (2.3) can be written as

$$
\left\{\begin{array}{l}
w_{i}^{\prime \prime}(t)+\lambda_{i} m^{2} r^{2(n-1)}(m(1-t)) a_{i}(r(m(1-t))) f_{i}(\mathbf{w}(t))=0, \quad 0<t<1 \\
w_{i}(1)=w_{i}(0)=0
\end{array}\right.
$$

where $i=1,2, \ldots, n, \mathbf{w}=\left(w_{1}, w_{2}, \ldots, w_{n}\right)^{T}$.

Letting $h_{i}(t)=m^{2} r^{2(n-1)}(m(1-t)) a_{i}(r(m(1-t)))$, then system (2.4) is equal to

$$
\left\{\begin{array}{l}
w_{i}^{\prime \prime}(t)+\lambda_{i} h_{i}(t) f_{i}(\mathbf{w})=0, \quad 0<t<1 \\
w_{i}(1)=w_{i}(0)=0
\end{array}\right.
$$

where $i=1,2, \ldots, n$.

The following conditions will be assumed throughout this paper:

$\left(H_{1}\right) h_{i}: J \rightarrow \mathcal{R}$ is continuous and there exists $0<\xi<1$ such that

$$
h_{i}(t) \geq 0, \quad t \in[0, \xi] ; \quad h_{i}(t) \leq 0, \quad t \in[\xi, 1], i=1,2, \ldots, n .
$$

Moreover, $h(t)$ does not vanish identically on any subintervals of $J$. 
$\left(H_{2}\right) f_{i}: \mathcal{R}_{+}^{n} \rightarrow \mathcal{R}_{+}$is continuous and there exists $0<c_{i} \leq 1$ such that

$$
f_{i}(\mathbf{w}) \geq c_{i} \varphi_{i}(\mathbf{w}), \quad i=1,2, \ldots, n,
$$

where $\varphi_{i}(\mathbf{w})=\max \left\{f_{i}(\mathbf{y}), 0 \leq y_{j} \leq w_{j}, j=1,2, \ldots, n\right\}$.

$\left(H_{3}\right)$ There exist $0<\theta_{i}<+\infty, \theta_{i} \neq 1$ and $k_{i}, l_{i}>0$ such that

$$
k_{i}\left(\sum_{i=1}^{n} w_{i}\right)^{\theta_{i}} \leq f_{i}(\mathbf{w}) \leq l_{i}\left(\sum_{i=1}^{n} w_{i}\right)^{\theta_{i}}, \quad \mathbf{w} \in \mathcal{R}_{+}^{n}, i=1,2, \ldots, n .
$$

$\left(H_{4}\right)$ There exists $0<\sigma<\xi$ such that

$$
c_{i}^{2} k_{i} \sigma^{\theta_{2}} \int_{\sigma}^{\xi} G(t, s) h_{i}^{+}(s) d s \geq l_{i} \xi^{\theta_{2}} \int_{\xi}^{1} G(t, s) h_{i}^{-}(s) d s, \quad i=1,2, \ldots, n .
$$

We define

$$
h_{i}^{+}(t)=\max \left\{h_{i}(t), 0\right\}, \quad h_{i}^{-}(t)=-\min \left\{h_{i}(t), 0\right\}, \quad i=1,2, \ldots, n .
$$

Then

$$
h_{i}(t)=h_{i}^{+}(t)-h_{i}^{-}(t), \quad i=1,2, \ldots, n .
$$

Next we give some lemmas which we will need later.

Lemma 2.1 (See [39]) By (2.1)-(2.4), system (1.1) admitting positive radial solutions is equal to system (2.4) having positive solutions.

Lemma 2.2 (Lemma 1 of [45]) Assume that $\left(H_{1}\right)-\left(H_{2}\right)$ hold. Then system (2.5) has a solution $\mathbf{w}=\left(w_{1}, \ldots, w_{i}, \ldots, w_{n}\right)$ given by

$$
w_{i}(t)=\lambda_{i} \int_{0}^{1} G(t, s) h_{i}(s) f_{i}(\mathbf{w}(s)) d s, \quad t \in J,
$$

where

$$
G(t, s)= \begin{cases}t(1-s), & 0 \leq t \leq s \leq 1 \\ s(1-t), & 0 \leq s \leq t \leq 1\end{cases}
$$

It is well known that $C[0,1]$ is a real Banach space with the norm given by $\|x\|_{\infty}=$ $\max _{t \in J}|x(t)|$. Let $X=\prod_{i=1}^{n} C[0,1]$, and for any $\mathbf{x}=\left(x_{1}, x_{2}, \ldots, x_{n}\right)^{T} \in X$,

$$
\|\mathbf{x}\|=\sum_{i=1}^{n}\left\|x_{i}\right\|_{\infty}
$$

Then $(X,\|\cdot\|)$ is a real Banach space. 
Define a cone $K$ in $X$ by

$$
K=\prod_{i=1}^{n} K_{i}
$$

where

$$
\begin{aligned}
& K_{i}=\left\{x_{i} \in C^{+}[0,1]: x_{i}(t) \text { is concave on }[0, \xi], \text { and convex on }[\xi, 1]\right\}, \quad i=1,2, \ldots, n, \\
& C^{+}[0,1]=\{x \in C[0,1]: x(t) \geq 0, \forall t \in J \text { and } x(0)=x(1)=0\} .
\end{aligned}
$$

We define some sets as follows:

$$
\begin{aligned}
& K_{i, r}=\left\{x_{i} \in K_{i} \mid\left\|x_{i}\right\|<r\right\}, \\
& K_{i, r, R}=\left\{x_{i} \in K_{i} \mid r<\left\|x_{i}\right\|<R\right\}, \\
& \partial K_{i, r}=\left\{x_{i} \in K_{i} \mid\left\|x_{i}\right\|=r\right\},
\end{aligned}
$$

where $R>r>0, i=1,2, \ldots, n$.

Lemma 2.3 (Proposition 2.1-2.2 of [16]) From (2.7), it is easy to verify that $G(t, s)$ has following properties:

(i) $G(t, s)>0, \forall t, s \in(0,1)$;

(ii) $G(t, s) \leq G(s, s), \forall t, s \in J$;

(iii) $G(t, s) \geq \sigma G(s, s), \forall t \in[\sigma, 1-\sigma], s \in J$, where $\sigma$ is defined in $\left(H_{4}\right)$.

We define the map $\mathbf{T}^{\boldsymbol{\Lambda}}: \mathcal{R}_{+}^{n} \rightarrow \mathcal{R}_{+}^{n}$ with components $\left(T_{1}^{\lambda_{1}}, \ldots, T_{i}^{\lambda_{i}}, \ldots, T_{n}^{\lambda_{n}}\right)$. Here, we understand $\mathbf{T}^{\boldsymbol{\Lambda}} \mathbf{w}=\left(T_{1}^{\lambda_{1}} \mathbf{w}, \ldots, T_{i}^{\lambda_{i}} \mathbf{w}, \ldots, T_{n}^{\lambda_{n}} \mathbf{w}\right)$, which

$$
\left(T_{i}^{\lambda_{i}} \mathbf{w}\right)(t)=\lambda_{i} \int_{0}^{1} G(t, s) h_{i}(s) f_{i}(\mathbf{w}(s)) d s, \quad t \in J, i=1,2, \ldots, n
$$

Cheng and Zhang [46] pointed out that the existence of a positive solutions of system (2.5) is equivalent to the existence of nontrivial fixed points of $\mathbf{T}^{\lambda}$ in $K$.

Lemma 2.4 Assume $\left(H_{1}\right)-\left(H_{4}\right)$ hold. Then $\mathbf{T}^{\Lambda}: K \rightarrow K$ is completely continuous.

Proof We show that $\mathbf{T}^{\boldsymbol{\Lambda}}(K) \subset K$, that is, for $\mathbf{w} \in K$, we show that $T_{i}^{\lambda_{i}} \in K_{i}, i=1,2, \ldots, n$. By (2.8), it is clear that $\left(T_{i}^{\lambda_{i}} \mathbf{w}\right)(0)=\left(T_{i}^{\lambda_{i}} \mathbf{w}\right)(1)=0(i=1,2, \ldots, n)$.

Define a function $q: J \rightarrow J$ as follows:

$$
q(t)=\min \left\{\frac{t}{\xi}, \frac{1-t}{1-\xi}\right\}
$$

Then $\min _{\sigma \leq t \leq \xi} q(t)=\frac{\sigma}{\xi}, \max _{\xi \leq t \leq 1} q(t)=1$.

For any $\mathbf{w} \in K$, we prove that

$$
\int_{0}^{1} G(t, s) h_{i}(s) f_{i}(\mathbf{w}(s)) d s \geq \int_{0}^{\sigma} G(t, s) h_{i}^{+}(s) f_{i}(\mathbf{w}(s)) d s .
$$


Since $\mathbf{w} \in K$ and $w_{i}(0)=w_{i}(1)=0, i=1,2, \ldots, n$, then we have

$$
\begin{aligned}
& \frac{w_{i}(t)-w_{i}(0)}{t-0} \geq \frac{w_{i}(\xi)-w_{i}(0)}{\xi-0}, \quad t \in[0, \xi] \Rightarrow w_{i}(t) \geq q(t) w_{i}(\xi), \quad t \in[0, \xi], \\
& \frac{w_{i}(t)-w_{i}(1)}{t-1} \geq \frac{w_{i}(\xi)-w_{i}(1)}{\xi-1}, \quad t \in[\xi, 1] \Rightarrow w_{i}(t) \leq q(t) w_{i}(\xi), \quad t \in[\xi, 1],
\end{aligned}
$$

where $i=1,2, \ldots, n$. Accordingly, we know that

$$
\mathbf{w}(t) \geq q(t) \mathbf{w}(\xi), \quad t \in[0, \xi] ; \quad \mathbf{w}(t) \leq q(t) \mathbf{w}(\xi), \quad t \in[\xi, 1]
$$

From the definition of $\varphi_{i}(i=1,2, \ldots, n)$, we also have

$$
\begin{array}{ll}
\varphi_{i}(\mathbf{w}(t)) \geq \varphi_{i}(q(t) \mathbf{w}(\xi)), & t \in[0, \xi], \\
\varphi_{i}(\mathbf{w}(t)) \leq \varphi_{i}(q(t) \mathbf{w}(\xi)), & t \in[\xi, 1] .
\end{array}
$$

Then, for $0 \leq t \leq 1$,

$$
\begin{array}{rl}
\int_{0}^{1} & G(t, s) h_{i}(s) f_{i}(\mathbf{w}(s)) d s-\int_{0}^{\sigma} G(t, s) h_{i}^{+}(s) f_{i}(\mathbf{w}(s)) d s \\
= & \int_{\sigma}^{\xi} G(t, s) h_{i}^{+}(s) f_{i}(\mathbf{w}(s)) d s-\int_{\xi}^{1} G(t, s) h_{i}^{-}(s) f_{i}(\mathbf{w}(s)) d s \\
\geq & c_{i} \int_{\sigma}^{\xi} G(t, s) h_{i}^{+}(s) \varphi_{i}(\mathbf{w}(s)) d s-\int_{\xi}^{1} G(t, s) h_{i}^{-}(s) \varphi_{i}(\mathbf{w}(s)) d s \\
\geq & c_{i} \int_{\sigma}^{\xi} G(t, s) h_{i}^{+}(s) \varphi_{i}(q(s) \mathbf{w}(\xi)) d s-\int_{\xi}^{1} G(t, s) h_{i}^{-}(s) \varphi_{i}(q(s) \mathbf{w}(\xi)) d s \\
\geq & c_{i} \int_{\sigma}^{\xi} G(t, s) h_{i}^{+}(s) f_{i}(q(s) \mathbf{w}(\xi)) d s-\frac{1}{c_{i}} \int_{\xi}^{1} G(t, s) h_{i}^{-}(s) f_{i}(q(s) \mathbf{w}(\xi)) d s \\
\geq & c_{i} \int_{\sigma}^{\xi} G(t, s) h_{i}^{+}(s) k_{i}\left(q(s) \sum_{i=1}^{n} w_{i}(\xi)\right)^{\theta_{i}} d s \\
\geq & 0 . \\
& -\frac{1}{c_{i}} \int_{\xi}^{1} G(t, s) h_{i}^{-}(s) l_{i}\left(q(s) \sum_{i=1}^{n} w_{i}(\xi)\right)^{\theta_{i}} d s \\
\geq & c_{i} k_{i}\left(\frac{\sigma}{\xi}\right)^{\theta_{i}}\left(\sum _ { i = 1 } ^ { n } l _ { i } \left(\sum_{i=1}^{n} w_{i}(\xi) w_{i}^{\theta_{i}} \int_{\sigma}^{\xi} G(t, s) h_{i}^{+}(s) d s\right.\right. \\
& \int_{\xi}^{1} G(t, s) h_{i}^{-}(s) d s \\
& \\
& \\
& \\
&
\end{array}
$$

Thus $T_{i}^{\lambda_{i}} \mathbf{w} \geq 0, i=1,2, \ldots, n$. 
By the above definitions and the properties of the Green's function $G(t, s)$, we have

$$
\begin{aligned}
& \left(T_{i}^{\lambda_{i}} \mathbf{w}\right)^{\prime \prime}(t)=\lambda_{i} h_{i}^{+}(t) f_{i}(\mathbf{w}(t)) \geq 0, \quad t \in[0, \xi], \\
& \left(T_{i}^{\lambda_{i}} \mathbf{w}\right)^{\prime \prime}(t)=-\lambda_{i} h_{i}^{-}(t) f_{i}(\mathbf{w}(t)) \leq 0, \quad t \in[0, \xi]
\end{aligned}
$$

where $i=1,2, \ldots, n$. Thus, $T_{i}^{\lambda_{i}} \mathbf{w} \in K_{i}$ and $T_{i}^{\lambda_{i}}(K) \subset K_{i}, i=1,2, \ldots, n$, that is, $\mathbf{T}^{\boldsymbol{\Lambda}}(K) \subset K$.

Similar to the proof of Lemma 2.4 in [47], one can prove $\mathbf{T}^{\boldsymbol{\Lambda}}: K \rightarrow K$ is completely continuous. The proof of Lemma 2.5 is complete.

The main proof is based on the well-known fixed point theorem of a cone expansion and a compression of norm type.

Lemma 2.5 (Theorem 2.3.4 of [48]) (Fixed point theorem of cone expansion and compression of norm type). Let $\Omega_{1}$ and $\Omega_{2}$ be two bounded open sets in a real Banach space $E$ such that $0 \in \Omega_{1}$ and $\bar{\Omega}_{1} \subset \Omega_{2}$. Let the operator $T: P \cap\left(\bar{\Omega}_{2} \backslash \Omega_{1}\right) \rightarrow$ P be completely continuous, where $P$ is a cone in E. Suppose that one of the two conditions

(i) $\|T x\| \leq\|x\|, \forall x \in P \cap \partial \Omega_{1}$ and $\|T x\| \geq\|x\|, \forall x \in P \cap \partial \Omega_{2}$ and

(ii) $\|T x\| \geq\|x\|, \forall x \in P \cap \partial \Omega_{1}$ and $\|T x\| \leq\|x\|, \forall x \in P \cap \partial \Omega_{2}$ is satisfied. Then $T$ has at least one fixed point in $P \cap\left(\bar{\Omega}_{2} \backslash \Omega_{1}\right)$.

\section{Main results}

Based on the lemmas mentioned above, we give the following theorems and their proofs.

Theorem 3.1 Assume that $\left(H_{1}\right)-\left(H_{4}\right)$ hold. If $\theta_{i}>1, i=1,2, \ldots, n$, then there exists $\lambda_{i, 0}>0$ such that system (1.1) has at least two positive radial solutions for $\lambda_{i} \in\left[\lambda_{i, 0},+\infty\right)$.

Proof On one hand, since $\theta_{i}>1$, by $\left(H_{3}\right)$, we get

$$
\lim _{\max _{1 \leq j \leq n} w_{j} \rightarrow 0} \frac{f_{i}(\mathbf{w})}{\sum_{i=1}^{n} w_{i}} \leq \lim _{\max _{1 \leq j \leq n} w_{j} \rightarrow 0} \frac{l_{i}\left(\sum_{i=1}^{n} w_{i}\right)^{\theta_{i}}}{\sum_{i=1}^{n} w_{i}}=0,
$$

where $i=1,2, \ldots, n$.

Furthermore, there exists a $r_{1}>0$ such that

$$
f_{i}(\mathbf{w}) \leq \varepsilon_{i} \sum_{i=1}^{n} w_{i}, \quad 0<w_{i}<r_{1}
$$

where $\varepsilon_{i}$ satisfies $\sum_{i=1}^{n} \max _{t \in J}\left(\lambda_{i} \varepsilon_{i} \int_{0}^{\xi} G(t, s) h_{i}^{+}(s) d s\right)<1, i=1,2, \ldots, n$.

Then, for any $w_{i} \in \partial K_{i, r_{1}}$, we have

$$
\begin{aligned}
\left\|T_{i}^{\lambda_{i}}(\mathbf{w})\right\|_{\infty} & =\max _{t \in J}\left|\lambda_{i} \int_{0}^{1} G(t, s) h_{i}(s) f_{i}(\mathbf{w}(s)) d s\right| \\
& =\max _{t \in J}\left|\lambda_{i} \int_{0}^{\xi} G(t, s) h_{i}^{+}(s) f_{i}(\mathbf{w}(s)) d s-\lambda_{i} \int_{\xi}^{1} G(t, s) h_{i}^{-}(s) f_{i}(\mathbf{w}(s)) d s\right| \\
& \leq \max _{t \in J}\left|\lambda_{i} \int_{0}^{\xi} G(t, s) h_{i}^{+}(s) f_{i}(\mathbf{w}(s)) d s\right|
\end{aligned}
$$




$$
\begin{aligned}
& \leq \max _{t \in J}\left|\lambda_{i} \int_{0}^{\xi} G(t, s) h_{i}^{+}(s) \varepsilon_{i} \sum_{i=1}^{n} w_{i}(s) d s\right| \\
& \leq \max _{t \in J} \lambda_{i} \int_{0}^{\xi} G(t, s) h_{i}^{+}(s) \varepsilon_{i}\left|\sum_{i=1}^{n} w_{i}(s)\right| d s \\
& \leq \max _{t \in J} \lambda_{i} \varepsilon_{i} \int_{0}^{\xi} G(t, s) h_{i}^{+}(s) d s\left(\sum_{i=1}^{n}\left\|w_{i}\right\|_{\infty}\right) \\
& =\max _{t \in J} \lambda_{i} \varepsilon_{i} \int_{0}^{\xi} G(t, s) h_{i}^{+}(s) d s\|\mathbf{w}\|,
\end{aligned}
$$

where $i=1,2, \ldots, n$.

Thus, for $w_{i} \in \partial K_{i, r_{1}}, i=1,2, \ldots, n$, we have

$$
\begin{aligned}
\left\|\mathbf{T}^{\boldsymbol{\Lambda}} \mathbf{w}\right\| & =\sum_{i=1}^{n}\left\|T_{i}^{\lambda_{i}} \mathbf{w}\right\|_{\infty} \\
& \leq \sum_{i=1}^{n} \max _{t \in J}\left(\lambda_{i} \varepsilon_{i} \int_{0}^{\xi} G(t, s) h_{i}^{+}(s) d s\right)\|\mathbf{w}\| \\
& <\|\mathbf{w}\| .
\end{aligned}
$$

We denote $\delta(t)=\min \left\{\frac{t}{\xi}, \frac{\xi-t}{\xi}\right\}, t \in[0, \xi]$. If $w_{i} \in K_{i}, i=1,2, \ldots, n$, then from the concave on $[0, \xi]$. So

$$
w_{i}(t) \geq \delta(t)\left\|w_{i}\right\|_{\infty}, \quad t \in[0, \xi], i=1,2, \ldots, n
$$

It follows that $w_{i}(t) \geq \alpha\left\|w_{i}\right\|_{\infty}, t \in\left[\frac{\sigma}{2}, \sigma\right]$, where $\alpha=\min _{\frac{\sigma}{2} \leq t \leq \sigma} \delta(t), i=1,2, \ldots, n$. Thus we have

$$
w_{i}(t) \geq \alpha\left\|w_{i}\right\|, \quad t \in\left[\frac{\sigma}{2}, \sigma\right], i=1,2, \ldots, n .
$$

Since $\theta_{i}>1, i=1,2, \ldots, n$, by $\left(H_{3}\right)$, we have

$$
\lim _{\min _{1 \leq j \leq n} w_{j} \rightarrow \infty} \frac{f_{i}(\mathbf{w})}{\sum_{i=1}^{n} w_{i}} \geq \lim _{\min _{1 \leq j \leq n} w_{j} \rightarrow \infty} \frac{k_{i}\left(\sum_{i=1}^{n} w_{i}\right)^{\theta_{i}}}{\sum_{i=1}^{n} w_{i}}=\infty,
$$

where $i=1,2, \ldots, n$.

Furthermore, there exists $0<r_{1}<R_{1}^{\prime}$ such that

$$
f_{i}(\mathbf{w}) \geq \eta_{i} \sum_{i=1}^{n} w_{i}, \quad w_{i} \geq R_{1}^{\prime}
$$

where $\eta_{i}$ satisfies $\sum_{i=1}^{n} \max _{t \in J}\left(\lambda_{i} \eta_{i} \alpha \int_{\frac{\sigma}{2}}^{\sigma} G(t, s) h_{i}^{+}(s) d s\right) \geq 1, i=1,2, \ldots, n$.

Choose $R_{1} \geq \frac{R_{1}^{\prime}}{\alpha}$. Then, for any $w_{i} \in \partial K_{i, R_{1}}, i=1,2, \ldots, n$, we have $\min \frac{\sigma}{2} \leq t \leq \sigma w_{i}(t) \geq$ $\min _{\frac{\sigma}{2} \leq t \leq \sigma} \delta(t)\left\|w_{i}\right\|_{\infty}=\alpha R_{1} \geq R_{1}^{\prime}$ and $f_{i}(\mathbf{w}(t)) \geq \eta_{i}\left(\sum_{i=1}^{n} w_{i}(t)\right), t \in\left[\frac{\sigma}{2}, \sigma\right], i=1,2, \ldots, n$.

Then, for $w_{i} \in \partial K_{i, R_{1}}$, we have

$$
\left\|T_{i}^{\lambda_{i}} \mathbf{w}\right\|_{\infty}=\max _{t \in J}\left|\lambda_{i} \int_{0}^{1} G(t, s) h_{i}(s) f_{i}(\mathbf{w}(s)) d s\right|
$$




$$
\begin{aligned}
& \geq \max _{t \in J}\left|\lambda_{i} \int_{0}^{\sigma} G(t, s) h_{i}^{+}(s) f_{i}(\mathbf{w}(s)) d s\right| \\
& \geq \max _{t \in J}\left|\lambda_{i} \int_{\frac{\sigma}{2}}^{\sigma} G(t, s) h_{i}^{+}(s) \eta_{i} \sum_{i=1}^{n} w_{i}(s) d s\right| \\
& \geq \max _{t \in J} \lambda_{i} \int_{\frac{\sigma}{2}}^{\sigma} G(t, s) h_{i}^{+}(s) \eta_{i} \alpha \sum_{i=1}^{n}\left\|w_{i}\right\|_{\infty} d s \\
& =\max _{t \in J} \lambda_{i} \eta_{i} \alpha \int_{\frac{\sigma}{2}}^{\sigma} G(t, s) h_{i}^{+}(s) d s\|\mathbf{w}\|,
\end{aligned}
$$

where $i=1,2, \ldots, n$.

Thus, for $w_{i} \in \partial K_{i, R_{1}}, i=1,2, \ldots, n$, we have

$$
\begin{aligned}
\left\|\mathbf{T}^{\boldsymbol{\Lambda}} \mathbf{w}\right\| & =\sum_{i=1}^{n}\left\|T_{i}^{\lambda_{i}} \mathbf{w}\right\|_{\infty} \\
& \geq \sum_{i=1}^{n} \max _{t \in J}\left(\lambda_{i} \eta_{i} \alpha \int_{\frac{\sigma}{2}}^{\sigma} G(t, s) h_{i}^{+}(s) d s\right)\|\mathbf{w}\| \\
& \geq\|\mathbf{w}\| .
\end{aligned}
$$

In addition, choose a number $0<r<r_{1}$. Noticing that $f_{i}(\mathbf{w})>0$ for all $\mathbf{w}>\mathbf{0}$, we can define

$$
f_{i, r}=\min \left\{f_{i}(\mathbf{w}): \alpha r \leq w_{i} \leq r\right\}, \quad i=1,2, \ldots, n
$$

Let $\lambda_{i, 0}=\frac{r}{\max _{t \in J} \int_{\frac{\sigma}{2}}^{\sigma} G(t, s) h_{i}^{+}(s) f_{i, r} d s}$.

If $w_{i} \in \partial K_{i, r}$, then $\left\|w_{i}\right\|_{\infty}=r$ and $\alpha r=\min _{\frac{\sigma}{2} \leq t \leq \sigma} \delta(t)\left\|w_{i}\right\|_{\infty} \leq w_{i}(t) \leq\left\|w_{i}\right\|_{\infty}=r, t \in$ $\left[\frac{\sigma}{2}, \sigma\right], i=1,2, \ldots, n$. It is clear that $f_{i}(\mathbf{w}(t)) \geq f_{i, r}, t \in\left[\frac{\sigma}{2}, \sigma\right], i=1,2, \ldots, n$. Then, for $w_{i} \in$ $\partial K_{i, r}$, we have

$$
\begin{aligned}
\left\|T_{i}^{\lambda_{i}} \mathbf{w}\right\|_{\infty} & =\max _{t \in J}\left|\lambda_{i} \int_{0}^{1} G(t, s) h_{i}(s) f_{i}(\mathbf{w}(s)) d s\right| \\
& \geq \max _{t \in J}\left|\lambda_{i} \int_{0}^{\sigma} G(t, s) h_{i}^{+}(s) f_{i}(\mathbf{w}(s)) d s\right| \\
& \geq \max _{t \in J} \lambda_{i} \int_{\frac{\sigma}{2}}^{\sigma} G(t, s) h_{i}^{+}(s) f_{i, r} d s,
\end{aligned}
$$

where $i=1,2, \ldots, n$.

Thus, for $w_{i} \in \partial K_{i, r}, i=1,2, \ldots, n$, we have

$$
\begin{aligned}
\left\|\mathbf{T}^{\boldsymbol{\Lambda}} \mathbf{w}\right\| & =\sum_{i=1}^{n}\left\|T_{i}^{\lambda_{i}} \mathbf{w}\right\|_{\infty} \\
& \geq \sum_{i=1}^{n} \max _{t \in J}\left(\lambda_{i} \int_{\frac{\sigma}{2}}^{\sigma} G(t, s) h_{i}^{+}(s) f_{i, r} d s\right) \\
& \geq \sum_{i=1}^{n} \max _{t \in J} \lambda_{i, 0}\left(\int_{\frac{\sigma}{2}}^{\sigma} G(t, s) h_{i}^{+}(s) f_{i, r} d s\right)
\end{aligned}
$$




$$
=n r=\sum_{i=1}^{n}\left\|w_{i}\right\|_{\infty}=\|\mathbf{w}\|
$$

Applying Lemma 2.5 to (3.1), (3.2) and (3.3) shows that $\mathbf{T}^{\boldsymbol{\Lambda}}$ admits at least two fixed points $\mathbf{w}_{1}, \mathbf{w}_{2}$, where $\mathbf{w}_{1} \in \bar{K}_{R_{1}} \backslash \bar{K}_{r_{1}}$ and $\mathbf{w}_{2} \in K_{r_{1}} \backslash K_{r}$. Thus it follows from Lemma 2.1 that, if $\theta_{i}>1, i=1,2, \ldots, n$, there exists $\lambda_{i, 0}>0$ such that system (1.1) has at least two positive radial solutions for $\lambda_{i} \in\left[\lambda_{i, 0},+\infty\right)$. This finishes the proof of Theorem 3.1.

Theorem 3.2 Assume that $\left(H_{1}\right)-\left(H_{4}\right)$ hold. If $0<\theta_{i}<1, i=1,2, \ldots, n$, then there exists $\lambda_{i}^{0}>0$ such that system (1.1) admits at least two positive radial solutions for $\lambda_{i} \in\left(0, \lambda_{i}^{0}\right], i=$ $1,2, \ldots, n$.

Proof On one hand, since $0<\theta_{i}<1$, by $\left(H_{3}\right)$, we get

$$
\lim _{\max _{1 \leq j \leq n} w_{j} \rightarrow 0} \frac{f_{i}(\mathbf{w})}{\sum_{i=1}^{n} w_{i}} \geq \lim _{\max _{1 \leq j \leq n} w_{j} \rightarrow 0} \frac{k_{i}\left(\sum_{i=1}^{n} w_{i}\right)^{\theta_{i}}}{\sum_{i=1}^{n} w_{i}}=\infty
$$

where $i=1,2, \ldots, n$.

Furthermore, there exists a $r_{2}>0$ such that

$$
f_{i}(\mathbf{w}) \geq \eta_{i}^{1} \sum_{i=1}^{n} w_{i}, \quad 0<w_{i}<r_{2}
$$

where $\eta_{i}^{1}$ satisfies $\sum_{i=1}^{n} \max _{t \in J} \lambda_{i} \eta_{i}^{1} \alpha \int_{\frac{\sigma}{2}}^{\sigma} G(t, s) h_{i}^{+}(s) d s>1, i=1,2, \ldots, n$. Thus $\min \left\{f_{i}(\mathbf{w})\right.$ : $\left.\alpha r_{2} \leq w_{i} \leq r_{2}, i=1,2, \ldots, n\right\} \geq \eta_{i}^{1} \sum_{i=1}^{n} w_{i}, i=1,2, \ldots, n$.

Then, for any $w_{i} \in \partial K_{i, r_{2}}, i=1,2, \ldots, n$, we have

$$
\begin{aligned}
\left\|T_{i}^{\lambda_{i}} \mathbf{w}\right\|_{\infty} & =\max _{t \in J}\left|\lambda_{i} \int_{0}^{1} G(t, s) h_{i}(s) f_{i}(\mathbf{w}(s)) d s\right| \\
& \geq \max _{t \in J}\left|\lambda_{i} \int_{0}^{\sigma} G(t, s) h_{i}(s) f_{i}(\mathbf{w}(s)) d s\right| \\
& \geq \max _{t \in J}\left|\lambda_{i} \int_{\frac{\sigma}{2}}^{\sigma} G(t, s) h_{i}^{+}(s) \eta_{i}^{1} \sum_{i=1}^{n} w_{i}(s) d s\right| \\
& \geq \max _{t \in J} \lambda_{i} \int_{\frac{\sigma}{2}}^{\sigma} G(t, s) h_{i}^{+}(s) \eta_{i}^{1} \alpha \sum_{i=1}^{n}\left\|w_{i}\right\|_{\infty} d s \\
& \geq \max _{t \in J} \lambda_{i} \eta_{i}^{1} \alpha \int_{\frac{\sigma}{2}}^{\sigma} G(t, s) h_{i}^{+}(s) d s\|\mathbf{w}\|,
\end{aligned}
$$

where $i=1,2, \ldots, n$.

Thus, for $w_{i} \in \partial K_{i, r_{2}}, i=1,2, \ldots, n$, we have

$$
\begin{aligned}
\left\|\mathbf{T}^{\mathbf{\Lambda}} \mathbf{w}\right\| & =\sum_{i=1}^{n}\left\|T_{i}^{\lambda_{i}} \mathbf{w}\right\|_{\infty} \\
& \geq \sum_{i=1}^{n} \max _{t \in J} \lambda_{i} \eta_{i}^{1} \alpha \int_{\frac{\sigma}{2}}^{\sigma} G(t, s) h_{i}^{+}(s) d s\|\mathbf{w}\|>\|\mathbf{w}\| .
\end{aligned}
$$


On the other hand, since $0<\theta_{i}<1$, by $\left(H_{3}\right)$, we have

$$
\lim _{\min _{1 \leq j \leq n} w_{j} \rightarrow \infty} \frac{f_{i}(\mathbf{w})}{\sum_{i=1}^{n} w_{i}} \leq \lim _{\min _{1 \leq j \leq n} w_{j} \rightarrow \infty} \frac{l_{i}\left(\sum_{i=1}^{n} w_{i}\right)^{\theta_{i}}}{\sum_{i=1}^{n} w_{i}}=0
$$

where $i=1,2, \ldots, n$.

Furthermore, there exists $0<r_{2}<R_{2}^{\prime}$ such that

$$
f_{i}(\mathbf{w}) \leq \varepsilon_{i}^{1}\left(\sum_{i=1}^{n} w_{i}\right), \quad w_{i} \geq R_{2}^{\prime},
$$

where $\varepsilon_{i}^{1}$ satisfies $\sum_{i=1}^{n} \max _{t \in J} \lambda_{i} \int_{0}^{\xi} G(t, s) h_{i}^{+}(s) \varepsilon_{i}^{1} d s \leq \frac{1}{2}, i=1,2, \ldots, n$.

Let $M_{i}=\max \left\{f_{i}(\mathbf{w}): 0 \leq w_{i} \leq R_{2}^{\prime}, i=1,2, \ldots, n\right\}$. It implies that

$$
f_{i}(\mathbf{w}) \leq \varepsilon_{i}^{1}\left(\sum_{i=1}^{n} w_{i}\right)+M_{i}, \quad x, y \geq 0, i=1,2, \ldots, n
$$

Choose $R_{2} \geq\left\{R_{2}^{\prime}, \frac{2}{n} \sum_{i=1}^{n} \max _{t \in J} \lambda_{i} \int_{0}^{\xi} G(t, s) h_{i}^{+}(s) M_{i} d s\right\}$. If $w_{i} \in \partial K_{i, R_{2}}$, then $\left\|w_{i}\right\|_{\infty}=R_{2}$ and $0 \leq w_{i}(t) \leq R_{2}, t \in J, i=1,2, \ldots, n$. It is easy to see that $f_{i}(\mathbf{w}(t)) \leq \varepsilon_{i}^{1}\left(\sum_{i=1}^{n} w_{i}(t)\right)+M_{i}, t \in$ $J, i=1,2, \ldots, n$. Then, for any $w_{i} \in \partial K_{i, R_{2}}$, we have

$$
\begin{aligned}
\left\|T_{i}^{\lambda_{i}} \mathbf{w}\right\|_{\infty} & =\max _{t \in J}\left|\lambda_{i} \int_{0}^{1} G(t, s) h_{i}(s) f_{i}(\mathbf{w}(s)) d s\right| \\
& =\max _{t \in J}\left|\lambda_{i} \int_{0}^{\xi} G(t, s) h_{i}^{+}(s) f_{i}(\mathbf{w}(s)) d s-\lambda_{i} \int_{\xi}^{1} G(t, s) h_{i}^{-}(s) f_{i}(\mathbf{w}(s)) d s\right| \\
& \leq \max _{t \in J}\left|\lambda_{i} \int_{0}^{\xi} G(t, s) h_{i}^{+}(s) f_{i}(\mathbf{w}(s)) d s\right| \\
& \leq \max _{t \in J}\left|\lambda_{i} \int_{0}^{\xi} G(t, s) h_{i}^{+}(s)\left[\varepsilon_{i}^{1} \sum_{i=1}^{n} w_{i}(s)+M_{i}\right] d s\right| \\
& \leq \max _{t \in J} \lambda_{i} \int_{0}^{\xi} G(t, s) h_{i}^{+}(s) \varepsilon_{i}^{1} \sum_{i=1}^{n}\left\|w_{i}\right\|_{\infty} d s+\max _{t \in J} \lambda_{i} \int_{0}^{\xi} G(t, s) h_{i}^{+}(s) M_{i} d s
\end{aligned}
$$

where $i=1,2, \ldots, n$.

Thus, for $w_{i} \in \partial K_{i, R_{2}}, i=1,2, \ldots, n$, we have

$$
\begin{aligned}
\left\|\mathbf{T}^{\boldsymbol{\Lambda}} \mathbf{w}\right\|= & \sum_{i=1}^{n}\left\|T_{i}^{\lambda_{i}} \mathbf{w}\right\|_{\infty} \\
\leq & \sum_{i=1}^{n}\left(\max _{t \in J} \lambda_{i} \int_{0}^{\xi} G(t, s) h_{i}^{+}(s) \varepsilon_{i}^{1} \sum_{i=1}^{n}\left\|w_{i}\right\|_{\infty} d s\right. \\
& \left.+\max _{t \in J} \lambda_{i} \int_{0}^{\xi} G(t, s) h_{i}^{+}(s) M_{i} d s\right) \\
\leq & \sum_{i=1}^{n} \max _{t \in J} \lambda_{i} \int_{0}^{\xi} G(t, s) h_{i}^{+}(s) \varepsilon_{i}^{1} d s\|\mathbf{w}\|+\sum_{i=1}^{n} \max _{t \in J} \lambda_{i} \int_{0}^{\xi} G(t, s) h_{i}^{+}(s) M_{i} d s
\end{aligned}
$$




$$
\begin{aligned}
& \leq \frac{1}{2}\|\mathbf{w}\|+\frac{n}{2} R_{2} \\
& =\frac{1}{2}\|\mathbf{w}\|+\frac{1}{2}\|\mathbf{w}\| \\
& =\|\mathbf{w}\| .
\end{aligned}
$$

In addition, choose a number $0<r^{\prime}<r_{1}$. Noticing that $f_{i}(\mathbf{w})>0$ for all $w_{i}>0$, we can define

$$
f_{i}^{r^{\prime}}=\max \left\{f_{i}(\mathbf{w}): 0<w_{j} \leq r^{\prime}, j=1,2, \ldots, n\right\},
$$

where $i=1,2, \ldots, n$.

Let $\lambda_{0}=\frac{r^{\prime}}{\max _{t \in J} \int_{0}^{\xi} G(t, s) h_{i}^{+}(s) f_{i}^{r^{\prime}} d s}$.

If $w_{i} \in \partial K_{r^{\prime}}$, then $\left\|w_{i}\right\|_{\infty}=r^{\prime}$ and $0 \leq w_{i}(t) \leq\left\|w_{i}\right\|_{\infty}=r^{\prime}, t \in J, i=1,2, \ldots, n$. It is clear that $f_{i}(\mathbf{w}(t)) \leq f_{i}^{r^{\prime}}, t \in J, i=1,2, \ldots, n$. Then, for $w_{i} \in \partial K_{i, r^{\prime}}$, we have

$$
\begin{aligned}
\left\|T_{i}^{\lambda_{i}} \mathbf{w}\right\|_{\infty} & =\max _{t \in J}\left|\lambda_{i} \int_{0}^{1} G(t, s) h_{i}(s) f_{i}(\mathbf{w}(s)) d s\right| \\
& =\max _{t \in J}\left|\lambda_{i} \int_{0}^{\xi} G(t, s) h_{i}^{+}(s) f_{i}(\mathbf{w}(s)) d s-\lambda_{i} \int_{\xi}^{1} G(t, s) h_{i}^{-}(s) f_{i}(\mathbf{w}(s)) d s\right| \\
& \leq \max _{t \in J}\left|\lambda_{i} \int_{0}^{\xi} G(t, s) h_{i}^{+}(s) f_{i}(\mathbf{w}(s)) d s\right| \\
& \leq \max _{t \in J}\left|\lambda_{i} \int_{0}^{\xi} G(t, s) h_{i}^{+}(s) f_{i}^{r^{\prime}} d s\right| \\
& \leq \max _{t \in J} \lambda_{i}^{0} \int_{0}^{\xi} G(t, s) h_{i}^{+}(s) f_{i}^{r^{\prime}} d s,
\end{aligned}
$$

where $i=1,2, \ldots, n$.

Thus, for $w_{i} \in \partial K_{i, r^{\prime}}, i=1,2, \ldots, n$, we have

$$
\begin{aligned}
\left\|\mathbf{T}^{\lambda} \mathbf{w}\right\| & =\sum_{i=1}^{n}\left\|T_{i}^{\lambda_{i}} \mathbf{w}\right\|_{\infty} \\
& \leq \sum_{i=1}^{n} \max _{t \in J} \lambda_{i}^{0} \int_{0}^{\xi} G(t, s) h_{i}^{+}(s) f_{i}^{r^{\prime}} d s \\
& =n r^{\prime}=\sum_{i=1}^{n}\left\|w_{i}\right\|_{\infty}=\|\mathbf{w}\| .
\end{aligned}
$$

Applying Lemma 2.5 to (3.4), (3.5) and (3.6) shows that $\mathbf{T}^{\boldsymbol{\Lambda}}$ admits at least two fixed points $\mathbf{w}^{1}, \mathbf{w}^{2}$, where $\mathbf{w}^{1} \in \bar{K}_{R_{2}} \backslash \bar{K}_{r_{2}}, \mathbf{w}^{2} \in K_{r_{2}} \backslash K_{r^{\prime}}$. Thus it follows from Lemma 2.1 that, if $0<\theta_{i}<1, i=1,2, \ldots, n$, there exists $\lambda_{i}^{0}>0$ such that system (1.1) has at least two positive radial solutions for $\lambda_{i} \in\left(0, \lambda_{i}^{0}\right]$. The proof of Theorem 3.2 is completed.

\section{Some special cases and an example}

In this part, we consider two special cases: $\boldsymbol{\Lambda} \equiv \mathbf{1}$ of system (1.1) and the weight function $\mathbf{a}(|\mathbf{x}|)$ is positive on $\left(R_{1}, R_{2}\right)$. 


\subsection{Case of $\Lambda \equiv 1$}

We consider $\boldsymbol{\Lambda} \equiv \mathbf{1}$, that is, $\lambda_{i} \equiv 1(i=1,2, \ldots, n)$. If $\boldsymbol{\Lambda} \equiv \mathbf{1}$, system (1.1) translates into the system (4.1):

$$
\left\{\begin{array}{l}
\Delta \mathbf{u}(\mathbf{x})+\mathbf{a}(|\mathbf{x}|) \mathbf{f}(\mathbf{u}(\mathbf{x}))=\mathbf{0}, \quad R_{1}<|\mathbf{x}|<R_{2} \\
\left.\mathbf{u}\right|_{|\mathbf{x}|=R_{1}}=\left.\mathbf{u}\right|_{|\mathbf{x}|=R_{2}}=\mathbf{0}
\end{array}\right.
$$

Similar to system (2.5), we transform system (4.1) into the system (4.2):

$$
\left\{\begin{array}{l}
w_{i}^{\prime \prime}(t)+h_{i}(t) f_{i}(\mathbf{w})=0, \quad 0<t<1 \\
w_{i}(1)=w_{i}(0)=0
\end{array}\right.
$$

We define the map $\mathbf{T}: R_{+}^{n} \rightarrow R_{+}^{n}$ with components $\left(T_{1}, \ldots, T_{i}, \ldots, T_{n}\right)$. Here, we understand $\mathbf{T w}=\left(T_{1} \mathbf{w}, \ldots, T_{i} \mathbf{w}, \ldots, T_{n} \mathbf{w}\right)$, where

$$
\left(T_{i} \mathbf{w}\right)(t)=\int_{0}^{1} G(t, s) h_{i}(s) f_{i}(\mathbf{w}(s)) d s, \quad i=1,2, \ldots, n .
$$

As Cheng and Zhang [46] pointed out, the existence of a positive solution of system (4.1) is equivalent to the existence of a nontrivial fixed point of $\mathbf{T}$ in $K$.

Lemma 4.1 Assume $\left(H_{1}\right)-\left(H_{4}\right)$ hold. $\mathbf{T}: K \rightarrow K$ is completely continuous.

Theorem 4.1 Assume $\left(H_{1}\right)-\left(H_{4}\right)$ hold. System (4.1) has at least one positive radial solution.

Proof We denote

$$
M=\left[\sum_{i=1}^{n} \int_{0}^{\sigma} \sigma G(s, s) h_{i}^{+}(s) d s\right]^{-1}, \quad N=\left[\sum_{i=1}^{n} \int_{0}^{\xi} G(s, s) h_{i}^{+}(s) d s\right]^{-1} .
$$

Let $\theta_{i}>1(i=1,2, \ldots, n)$. On the one hand, since $\theta_{i}>1(i=1,2, \ldots, n)$, by $\left(H_{3}\right)$, we have

$$
\lim _{\min _{1 \leq j \leq n} w_{j} \rightarrow 0} \frac{f_{i}(\mathbf{w})}{\sum_{i=1}^{n} w_{i}} \leq \lim _{\min _{1 \leq j \leq n} w_{j} \rightarrow 0} \frac{l_{i}\left(\sum_{i=1}^{n} w_{i}\right)^{\theta_{i}}}{\sum_{i=1}^{n} w_{i}}=0 .
$$

Furthermore, there exists a $r_{1}>0$ such that

$$
f_{i}(\mathbf{w}) \leq N \sum_{i=1}^{n} w_{i}, \quad 0<w_{i}<r_{1}, i=1,2, \ldots, n
$$

If $w_{i} \in \partial K_{i, r}$, then $\left\|w_{i}\right\|_{\infty}=r_{1}$ and $0 \leq w_{i}(t) \leq\left\|w_{i}\right\|_{\infty}=r_{1}, t \in J, i=1,2, \ldots, n$. This implies that $f_{i}(\mathbf{w}(t)) \leq N \sum_{i=1}^{n} w_{i}(t) \leq N n r_{1}, t \in J, i=1,2, \ldots, n$. Then, for any $w_{i} \in \partial K_{i, r_{1}}(i=$ $1,2, \ldots, n)$, we have

$$
\|\mathbf{T w}\|=\sum_{i=1}^{n}\left\|T_{i} \mathbf{w}\right\|_{\infty}=\sum_{i=1}^{n} \max _{t \in J}\left|\int_{0}^{1} G(t, s) h_{i}(s) f_{i}(\mathbf{w}(s)) d s\right|
$$




$$
\begin{aligned}
& =\sum_{i=1}^{n} \max _{t \in J}\left|\int_{0}^{\xi} G(t, s) h_{i}^{+}(s) f_{i}(\mathbf{w}(s)) d s-\int_{\xi}^{1} G(t, s) h_{i}^{-}(s) f_{i}(\mathbf{w}(s)) d s\right| \\
& \leq \sum_{i=1}^{n} \max _{t \in J}\left|\int_{0}^{\xi} G(t, s) h_{i}^{+}(s) f_{i}(\mathbf{w}(s)) d s\right| \\
& \leq \sum_{i=1}^{n}\left|\int_{0}^{\xi} G(s, s) h_{i}^{+}(s) f_{i}(\mathbf{w}(s)) d s\right| \\
& \leq \sum_{i=1}^{n} \int_{0}^{\xi} G(s, s) h_{i}^{+}(s) N n r_{1} d s \\
& =n r_{1}=\|\mathbf{w}\| .
\end{aligned}
$$

Consequently,

$$
\|\mathbf{T w}\| \leq\|\mathbf{w}\|, \quad \forall \mathbf{w} \in \partial K_{r_{1}} .
$$

On the other hand, if $w_{i} \in K_{i}, i=1,2, \ldots, n$, then from the concavity on $[0, \xi]$,

$$
w_{i}(t) \geq \delta(t)\left\|w_{i}\right\|_{\infty}, \quad t \in[0, \xi], i=1,2, \ldots, n
$$

It follows that $w_{i}(t) \geq \alpha\left\|w_{i}\right\|_{\infty}, t \in\left[\frac{\sigma}{2}, \sigma\right]$, where $\alpha=\min _{\frac{\sigma}{2} \leq t \leq \sigma} \delta(t), i=1,2, \ldots, n$. Thus we have

$$
w_{i}(t) \geq \alpha\left\|w_{i}\right\|, \quad t \in\left[\frac{\sigma}{2}, \sigma\right], i=1,2, \ldots, n
$$

Since $\theta_{i}>1, i=1,2, \ldots, n$, by $\left(H_{3}\right)$, we have

$$
\lim _{\min _{1 \leq j \leq n} w_{j} \rightarrow \infty} \frac{f_{i}(\mathbf{w})}{\sum_{i=1}^{n} w_{i}} \geq \lim _{\min _{1 \leq j \leq n} w_{j} \rightarrow \infty} \frac{k_{i}\left(\sum_{i=1}^{n} w_{i}\right)^{\theta_{i}}}{\sum_{i=1}^{n} w_{i}}=\infty .
$$

Furthermore, there exists $0<r_{1}<R_{1}^{\prime}<+\infty$ such that

$$
f_{i}(\mathbf{w}) \geq \frac{M \sum_{i=1}^{n} w_{i}}{\alpha}, \quad w_{i} \geq R_{1}^{\prime}, i=1,2, \ldots, n
$$

Choose $R_{1} \geq \frac{R_{1}^{\prime}}{\alpha}$. Then, for any $w_{i} \in \partial K_{i, R_{1}}, i=1,2, \ldots, n$, we have $\min \frac{\sigma}{2} \leq t \leq \sigma w_{i}(t) \geq$

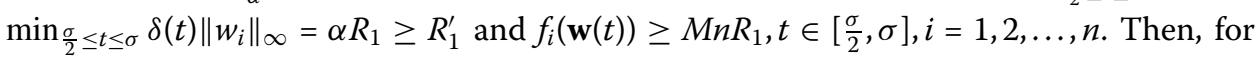
$w_{i} \in \partial K_{i, R_{1}}$, we have

$$
\begin{aligned}
\|\mathbf{T w}\| & =\sum_{i=1}^{n}\left\|T_{i} \mathbf{w}\right\|_{\infty}=\sum_{i=1}^{n} \max _{t \in J}\left|\int_{0}^{1} G(t, s) h_{i}(s) f_{i}(\mathbf{w}(s)) d s\right| \\
& \geq \sum_{i=1}^{n} \max _{t \in[\sigma, 1-\sigma]}\left|\int_{0}^{\sigma} G(t, s) h_{i}^{+}(s) f_{i}(\mathbf{w}(s)) d s\right| \\
& \geq \sum_{i=1}^{n} \int_{0}^{\sigma} \sigma G(s, s) h_{i}^{+}(s) f_{i}(\mathbf{w}(s)) d s
\end{aligned}
$$




$$
\geq \sum_{i=1}^{n} \int_{\frac{\sigma}{2}}^{\sigma} \sigma G(s, s) h_{i}^{+}(s) M n R_{1} d s=n R_{1}=\|\mathbf{w}\| .
$$

Consequently,

$$
\|\mathbf{T w}\| \geq\|\mathbf{w}\|, \quad \forall \mathbf{w} \in \partial K_{R_{1}} .
$$

Summing up we can show that $\mathbf{T}$ has at least one fixed point $\mathbf{w}_{1}$, where $\mathbf{w}_{1} \in \bar{K}_{R_{1}} \backslash \bar{K}_{r_{1}}$ by applying Lemma 2.5 to (4.4) and (4.5). According to Lemma 2.1, if $\theta_{i}>1, i=1,2, \ldots, n$, system (4.1) has at least one positive solution.

If $0<\theta_{i}<1(i=1,2, \ldots, n)$, the proof is similar. We omit it.

The proof of Theorem 4.1 is completed.

If $\min _{1 \leq i \leq n} \frac{l_{i}}{k_{i}}$ is sufficiency large, we have the following theorem.

Theorem 4.2 Assume that $\left(H_{1}\right)-\left(H_{4}\right)$ hold and there exist two positive numbers $A_{1}, B_{1}$ such that one of the following conditions is satisfied:

(i) $0<\theta_{i}<1, A_{1}<B_{1}$ and

$$
\begin{aligned}
& \max \left\{f_{i}(\mathbf{w}): 0 \leq w_{j} \leq A_{1}, j=1,2, \ldots, n\right\}<A_{1} M, \\
& \min \left\{f_{i}(\mathbf{w}): \alpha B_{1} \leq w_{j} \leq B_{1}, j=1,2, \ldots, n\right\}>B_{1} N .
\end{aligned}
$$

(ii) $1<\theta_{i}<+\infty, A_{1}>B_{1}$ and

$$
\begin{aligned}
& \min \left\{f_{i}(\mathbf{w}): \alpha B_{1} \leq w_{j} \leq B_{1}, j=1,2, \ldots, n\right\}>B_{1} N, \\
& \max \left\{f_{i}(\mathbf{w}): 0 \leq w_{j} \leq A_{1}, j=1,2, \ldots, n\right\}<A_{1} M .
\end{aligned}
$$

Then system (4.1) has at least three positive radial solutions.

Proof It is enough to prove the case (i).

We have the following claim.

Claim 4.1 If there exist two different positive numbers $A, B$ such that

$$
\begin{aligned}
& \max \left\{f_{i}(\mathbf{w}): \mathbf{0} \leq w_{j} \leq A, j=1,2, \ldots, n\right\} \leq A M, \\
& \min \left\{f_{i}(\mathbf{w}): \alpha B \leq w_{j} \leq B, j=1,2, \ldots, n\right\} \geq B N,
\end{aligned}
$$

then the operator $\mathbf{T}$ has one fixed point $\mathbf{w}^{*} \in K$ and $\min \{n A, n B\} \leq\left\|\mathbf{w}^{*}\right\| \leq \max \{n A, n B\}$.

The proof of Claim 4.1 is similar to the proof of Theorem 4.1.

Now, $\lim _{\max _{1 \leq j \leq n} w_{j} \rightarrow 0} \frac{f_{i}(\mathbf{w})}{\sum_{j=1}^{n} w_{j}}=+\infty$ and $\lim _{\min _{1 \leq j \leq n} w_{j} \rightarrow+\infty} \frac{f_{i}(\mathbf{w})}{\sum_{j=1}^{n} w_{j}}=0, i=1,2, \ldots, n$. By the proof of Theorem 4.1, we assert that there exist positive numbers $A_{2}, B_{2}$ such that $B_{2}<$ $A_{1}<B_{1}<A_{2}$ and

$$
\begin{aligned}
& \min \left\{f_{i}(\mathbf{w}): \alpha B_{2} \leq w_{j} \leq B_{2}, j=1,2, \ldots, n\right\}>B_{2} N, \\
& \max \left\{f_{i}(\mathbf{w}): \mathbf{0} \leq w_{j} \leq A_{2} j=1,2, \ldots, n\right\}<A_{2} M,
\end{aligned}
$$


where $i=1,2, \ldots, n$.

On the other hand, letting $\psi_{i}(\mathbf{w})=\min \left\{f_{i}(\mathbf{y}): \alpha \mathbf{w} \leq \mathbf{y} \leq \mathbf{w}\right\}(i=1,2, \ldots, n)$, then $\varphi_{i}, \psi_{i}$ : $\mathcal{R}_{+}^{n} \rightarrow \mathcal{R}_{+}(i=1,2, \ldots, n)$ are continuous. Since $M<N, \psi_{i}\left(\mathbf{B}_{2}\right)>B_{2} N, \varphi_{i}\left(\mathbf{A}_{2}\right)<A_{2} M$, we assert that there exist

$$
B_{2}<A_{1}^{\prime}<A_{1}<A_{1}^{\prime \prime}<B_{1}^{\prime}<B_{1}<B_{1}^{\prime \prime}<A_{2}
$$

such that

$$
\begin{aligned}
& \max \left\{f_{i}(\mathbf{w}): 0 \leq w_{j} \leq A_{1}^{\prime}, j=1,2, \ldots, n\right\}=\varphi_{i}\left(\mathbf{A}_{1}^{\prime}\right)<A_{1}^{\prime} M, \\
& \max \left\{f_{i}(\mathbf{w}): 0 \leq w_{j} \leq A_{1}^{\prime \prime}, j=1,2, \ldots, n\right\}=\varphi_{i}\left(\mathbf{A}_{1}^{\prime \prime}\right)<A_{1}^{\prime \prime} M, \\
& \min \left\{f_{i}(\mathbf{w}): \alpha B_{1}^{\prime} \leq w_{j} \leq B_{1}^{\prime}, j=1,2, \ldots, n\right\}=\psi_{i}\left(\mathbf{B}_{1}^{\prime}\right)>B_{1}^{\prime} N, \\
& \min \left\{f_{i}(\mathbf{w}): \alpha B_{1}^{\prime \prime} \leq w_{j} \leq B_{1}^{\prime \prime}, j=1,2, \ldots, n\right\}=\psi_{i}\left(\mathbf{B}_{1}^{\prime \prime}\right)>B_{1}^{\prime \prime} N,
\end{aligned}
$$

where

$$
\begin{array}{ll}
\mathbf{A}_{2}=(\underbrace{A_{2}, \ldots, A_{2}, \ldots, A_{2}}_{n})^{T}, & \mathbf{B}_{2}=(\underbrace{B_{2}, \ldots, B_{2}, \ldots, B_{2}}_{n})^{T}, \\
A_{1}^{\prime}=(\underbrace{A_{1}^{\prime}, \ldots, A_{1}^{\prime}, \ldots, A_{1}^{\prime}}_{n})^{T}, & A_{1}^{\prime \prime}=(\underbrace{A_{1}^{\prime \prime}, \ldots, A_{1}^{\prime \prime}, \ldots, A_{1}^{\prime \prime}}_{n})^{T}, \\
B_{1}^{\prime}=(\underbrace{B_{1}^{\prime}, \ldots, B_{1}^{\prime}, \ldots, B_{1}^{\prime}}_{n})^{T}, & B_{1}^{\prime \prime}=(\underbrace{B_{1}^{\prime \prime}, \ldots, B_{1}^{\prime \prime}, \ldots, B_{1}^{\prime \prime}}_{n})^{T} .
\end{array}
$$

By using Claim 4.1 for $\left(B_{2}, A_{1}^{\prime}\right),\left(A_{1}^{\prime \prime}, B_{1}^{\prime}\right),\left(B_{1}^{\prime \prime}, A_{2}\right)$, respectively, we see that the operator $\mathbf{T}$ has three fixed points $\mathbf{w}_{1}, \mathbf{w}_{2}, \mathbf{w}_{3} \in K$ satisfying

$$
n B_{2} \leq\left\|\mathbf{w}_{1}\right\| \leq n A_{1}^{\prime}<n A_{1}^{\prime \prime} \leq\left\|\mathbf{w}_{2}\right\| \leq n B_{1}^{\prime}<n B_{1}^{\prime \prime} \leq\left\|\mathbf{w}_{3}\right\| \leq n A_{2} .
$$

By Lemma 2.1 and Lemma 2.5 we know that system (4.1) has at least three positive radial solutions. The proof of Theorem 4.1 is completed.

\subsection{Case of definite weight function}

We consider the multiplicity of elliptic system (1.1) with definite function. By a series of transformations, (1.1) is transformed to (2.4). Assume the following conditions throughout:

$\left(A_{1}\right) \quad \boldsymbol{\Lambda}=\left(\lambda_{1}, \ldots, \lambda_{i}, \ldots, \lambda_{n}\right)>\mathbf{0}$ is a parameter vector;

$\left(A_{2}\right) h_{i} \in L^{1}[0,1]$ and there exists $\eta_{i}>0$ such that $h_{i}(t) \geq \eta_{i}$ a.e. on $J$.

$\left(A_{3}\right) f_{i}: \mathcal{R}_{+}^{n} \rightarrow \mathcal{R}_{+}$is continuous with $f_{i}(\mathbf{w})>0$ for $\mathbf{w}>\mathbf{0}$;

where $i=1,2, \ldots, n$.

Lemma 4.2 Let $G(t, s)$ be given as (2.6) and $0<\rho<\frac{1}{2}$. Then we have

$$
G(t, s) \geq \rho G(s, s), \quad t \in J_{\rho}, s \in J,
$$

where $J_{\rho}=[\rho, 1-\rho]$. 
Proof For $t \in J_{\rho}$ and $s \in J$, we have

$$
\begin{aligned}
& \frac{G(t, s)}{G(s, s)}=\frac{t(1-s)}{s(1-s)}=\frac{t}{s} \geq \rho, \quad t \leq s, \\
& \frac{G(t, s)}{G(s, s)}=\frac{s(1-t)}{s(1-s)}=\frac{1-t}{1-s} \geq \rho, \quad s \leq t .
\end{aligned}
$$

Then the proof is complete.

We define a cone $K$ in $X$ by

$$
K=\prod_{i=1}^{n} K_{i}
$$

where

$$
K_{i}=\left\{x_{i} \in C^{+}[0,1]: x_{i} \geq 0 \text { for } J, \min _{t \in J_{\rho}} x_{i}(t) \geq \rho\left\|x_{i}\right\|_{\infty}\right\}, \quad i=1,2, \ldots, n .
$$

Definition The map $\beta$ is said to be a nonnegative continuous concave function on a cone $K$ of a real Banach space $E$ if $\beta: K \rightarrow K$ is continuous and

$$
\beta(t x+(1-t) y) \geq t \beta(x)+(1-t) \beta(y)
$$

for all $x, y \in K$ and $t \in J$.

Let $K$ be a cone in a Banach space $X$. For positive numbers $0<c<d$, we define the convex set $K(\beta, c, d)$ by

$$
K(\beta, c, d)=\{x: x \in K, c \leq \beta(x),\|x\| \leq d\}
$$

In this part, $\beta(\mathbf{x})=\sum_{i=1}^{n} \beta_{i}\left(x_{i}\right)$, and we understand $K(\beta, c, d)=\left(K_{1}\left(\beta_{1}, c, d\right), K_{2}\left(\beta_{2}, c, d\right), \ldots\right.$, $\left.K_{n}\left(\beta_{n}, c, d\right)\right)$, where $K_{i}\left(\beta_{i}, c, d\right)=\left\{x_{i}: x_{i} \in K_{i}, c \leq \beta_{i}\left(x_{i}\right),\left\|x_{i}\right\|_{\infty} \leq d\right\}, i=1,2, \ldots, n$.

We define the map $\mathbf{T}^{\boldsymbol{\Lambda}}: K \rightarrow X$ with components $\left(T_{1}^{\lambda_{1}}, T_{2}^{\lambda_{2}}, \ldots, T_{n}^{\lambda_{n}}\right)^{T}$. Hence, we understand $\mathbf{T}^{\boldsymbol{\Lambda}} \mathbf{w}=\left(T_{1}^{\lambda_{1}} \mathbf{w}, T_{2}^{\lambda_{2}} \mathbf{w}, \ldots, T_{n}^{\lambda_{n}} \mathbf{w}\right)^{T}$, which

$$
\left(T_{i}^{\lambda_{i}} \mathbf{w}\right)(t)=\lambda_{i} \int_{0}^{1} G(t, s) h_{i}(s) f_{i}(\mathbf{w}(s)) d s, \quad t \in J, i=1,2, \ldots, n
$$

As Cheng and Zhang [46] pointed out, $\mathbf{w}$ is a positive radial solution of system (1.1) if and only if $\mathbf{w} \in K$ is a positive point of $\mathbf{T}^{\boldsymbol{\Lambda}}$.

Lemma 4.3 Suppose that $\left(A_{1}\right)-\left(A_{3}\right)$ hold. Then $\mathbf{T}^{\boldsymbol{\Lambda}}: K \rightarrow K$ is completely continuous.

Proof We just prove $T_{i}^{\lambda_{i}}: K \rightarrow K_{i}$ is completely continuous. For all $\mathbf{w} \in K, T_{i}^{\lambda_{i}} \mathbf{w} \geq 0$ on $J$ and

$$
\left\|T_{i}^{\lambda_{i}} \mathbf{w}\right\|_{\infty}=\max _{t \in J}\left|\lambda_{i} \int_{0}^{1} G(t, s) h_{i}(s) f_{i}(\mathbf{x}(s)) d s\right|
$$




$$
\leq \max _{t \in J}\left|\lambda_{i} \int_{0}^{1} G(s, s) h_{i}(s) f_{i}(\mathbf{x}(s)) d s\right|
$$

From Lemma 4.2, we have

$$
\begin{aligned}
\min _{t \in J_{\rho}} T_{i}^{\lambda_{i}} \mathbf{w}(t) & =\min _{t \in J_{\rho}} \lambda_{i} \int_{0}^{1} G(t, s) h_{i}(s) f_{i}(\mathbf{w}(s)) d s \\
& \geq \min _{t \in J_{\rho}} \lambda_{i} \int_{0}^{1} \rho G(s, s) h_{i}(s) f_{i}(\mathbf{w}(s)) d s \\
& \geq \min _{t \in J} \lambda_{i} \int_{0}^{1} \rho G(s, s) h_{i}(s) f_{i}(\mathbf{w}(s)) d s \\
& \geq\left\|T_{i}^{\lambda_{i}} \mathbf{w}\right\|_{\infty} .
\end{aligned}
$$

Thus, we have $T_{i}^{\lambda_{i}}(K) \subset K_{i}$, therefore $\mathbf{T}^{\boldsymbol{\Lambda}}(K) \subset K$.

Finally, from the standard process, it follows that $T_{i}^{\lambda_{i}}: K \rightarrow K_{i}$ is completely continuous, that is, $\mathbf{T}^{\boldsymbol{\Lambda}}: K \rightarrow K$ is completely continuous.

Lemma 4.4 (Leggett-Williams fixed point theorem) Let $K$ be a cone in a real Banach space $E, A: \bar{K}_{a} \rightarrow \bar{K}_{a}$ be completely continuous and $\beta$ be a nonnegative continuous concave functional on $K$ with $\beta(x) \leq\|x\|$ for all $x \in K_{a}$. Suppose there exist $0<d<a<b \leq c$ such that

(i) $\{x \in K(\beta, a, b): \beta(x)>a\} \neq \emptyset$ and $\beta(A x)>a$ for $x \in K(\beta, a, b)$;

(ii) $\|A x\|<d$ for $\|x\| \leq d$;

(iii) $\beta(A x)>a$ for $x \in K(\beta, a, c)$ with $\|A x\|>b$.

Then $A$ has at least three positive solutions $x_{1}, x_{2}, x_{3}$ satisfying

$$
\left\|x_{1}\right\|<d, \quad a<\beta\left(x_{2}\right), \quad\left\|x_{3}\right\|>d, \quad \beta\left(x_{3}\right)<a .
$$

Next, we begin by introducing the notation

$$
\begin{aligned}
& f_{i}^{0}=\limsup _{\|w\| \rightarrow 0^{+}} \frac{f_{i}(\mathbf{w})}{\|\mathbf{x}\|}, \quad f_{i}^{\infty}=\limsup _{\|w\| \rightarrow+\infty} \frac{f_{i}(\mathbf{w})}{\|\mathbf{x}\|}, \\
& f_{i, 0}=\liminf _{\|w\| \rightarrow 0^{+}} \frac{f_{i}(\mathbf{w})}{\|\mathbf{x}\|}, \quad f_{i, \infty}=\liminf _{\|w\| \rightarrow+\infty} \frac{f_{i}(\mathbf{w})}{\|\mathbf{x}\|}, \\
& f_{i}^{*}(l)=\min \left\{f_{i}(\mathbf{w}), 0 \leq\|\mathbf{w}\| \leq l\right\}, \quad f_{i}^{* *}(l)=\max \left\{f_{i}(\mathbf{w}), 0 \leq\|\mathbf{w}\| \leq l\right\}, \\
& N_{i}=\frac{1}{6} \lambda_{i} \rho \eta_{i}, \quad D_{i}=\lambda_{i} \int_{0}^{1} G(s, s) h_{i}(s) d s,
\end{aligned}
$$

where $i=1,2, \ldots, n$.

Theorem 4.3 Assume $\left(A_{1}\right)-\left(A_{3}\right)$ hold. For $\lambda_{i}>0$ there exist constants $0<M<C<\frac{C}{\rho}<L$ such that

$$
\begin{aligned}
& \qquad\left(A_{4}\right) f_{i}^{\infty}<\frac{1}{N_{i}}, \\
& \quad\left(A_{5}\right) f_{i}(\mathbf{w})<\frac{M}{D_{i}}, t \in J_{\rho}, \rho M \leq\left\|w_{i}\right\|_{\infty} \leq M \text {, and } f_{i}(\mathbf{w})>\frac{C}{N_{i}}, t \in J_{\rho}, C \leq\left\|w_{i}\right\|_{\infty} \leq \frac{C}{\rho} \text {, } \\
& \text { where } i=1,2, \ldots, n \text {. }
\end{aligned}
$$


Then system (1.1) has at least three positive solutions $\mathbf{w}_{1}, \mathbf{w}_{2}, \mathbf{w}_{3}$ satisfying

$$
\left\|\mathbf{w}_{\mathbf{1}}\right\|<n M, \quad n C<\beta\left(\mathbf{w}_{\mathbf{2}}\right), \quad\left\|\mathbf{w}_{\mathbf{3}}\right\|>n M, \quad \beta\left(\mathbf{w}_{\mathbf{3}}\right)<n C .
$$

Proof Let $\beta(\mathbf{w})=\sum_{i=1}^{n} \beta_{i}\left(w_{i}\right), \beta_{i}\left(w_{i}\right)=\min _{t \in J_{\rho}} w_{i}(t)$. It is clear that $\beta(\mathbf{w})$ is a nonnegative continuous concave functional on the cone $K$ satisfying $\beta(\mathbf{w}) \leq\|\mathbf{w}\|$ for all $\mathbf{w} \in K$.

By $\left(A_{4}\right)$ there exist $0<\varepsilon<\frac{1}{N_{i}}$ such that

$$
f_{i}(\mathbf{w}) \leq \varepsilon\left\|w_{i}\right\|_{\infty}, \quad\left\|w_{i}\right\|_{\infty}>r
$$

By the definition of $f_{i}^{* *}$, we have

$$
0 \leq f_{i}(\mathbf{w}) \leq \varepsilon\left\|w_{i}\right\|_{\infty}+f_{i}^{* *}, \quad\left\|w_{i}\right\|_{\infty}>0 .
$$

Let $L>\max \left\{\frac{f^{* *}}{\max _{t \in J} \lambda_{i} \int_{0}^{1} G(t, s) h_{i}^{+}(s) \varepsilon_{i} d s}\right\}$. Then, for $\mathbf{w} \in \bar{K}_{n L}$,

$$
\begin{aligned}
\left\|\mathbf{T}^{\boldsymbol{\Lambda}} \mathbf{w}\right\| & =\sum_{i=1}^{n}\left\|T_{i}^{\lambda} \mathbf{w}\right\|_{\infty}=\sum_{i=1}^{n} \max _{t \in J}\left|\lambda_{i} \int_{0}^{1} G(t, s) h_{i}(s) f_{i}(\mathbf{w}(s)) d s\right| \\
& \leq \sum_{i=1}^{n} \max _{t \in J}\left|\lambda_{i} \int_{0}^{1} G(t, s) h_{i}^{+}(s) f_{i}(\mathbf{w}(s)) d s\right| \\
& \leq \sum_{i=1}^{n} \max _{t \in J} \lambda_{i} \int_{0}^{1} G(t, s) h_{i}^{+}(s)\left(\varepsilon_{i} \mathbf{w}(s)+f_{i}^{* *}\right) d s \\
& \leq \sum_{i=1}^{n} \max _{t \in J} \lambda_{i} \int_{0}^{1} G(t, s) h_{i}^{+}(s)\left(\varepsilon_{i}\|\mathbf{w}\|+f_{i}^{* *}\right) d s \\
& =\sum_{i=1}^{n} \max _{t \in J} \lambda_{i} \int_{0}^{1} G(t, s) h_{i}^{+}(s)\left(\varepsilon_{i} L+f_{i}^{* *}\right) d s \\
& <n L,
\end{aligned}
$$

which implies that $\mathbf{T}^{\boldsymbol{\Lambda}} \mathbf{w} \in K_{n L}$. Hence, we have shown that the map $\mathbf{T}^{\boldsymbol{\Lambda}}: \bar{K}_{n L} \rightarrow \bar{K}_{n L}$ is completely continuous.

Next, we verify that $\left\{\mathbf{x}: \mathbf{x} \in K\left(\beta, n C, \frac{n C}{\rho}\right), \beta(\mathbf{x})>n C\right\} \neq \emptyset$ and $\beta\left(\mathbf{T}^{\Lambda} \mathbf{x}\right)>n C$ for all $\mathbf{x} \in$ $K\left(\beta, n C, \frac{n C}{\rho}\right)$.

Take $\mathbf{w}_{0}=\left(w_{1}^{0}(t), w_{2}^{0}(t), \ldots, w_{n}^{0}(t)\right), w_{i}^{0}=\frac{C}{\rho}, i=1,2, \ldots, n$, for $t \in J$. Then

$$
\mathbf{w}_{0}=\left\{\mathbf{w}: \mathbf{w} \in K\left(\beta, n C, \frac{n C}{\rho}\right), \beta(\mathbf{w})>n C\right\}
$$

which shows that

$$
\left\{\mathbf{w}: \mathbf{w} \in K\left(\beta, n C, \frac{n C}{\rho}\right), \beta(\mathbf{w})>n C\right\} \neq \emptyset
$$


For all $\mathbf{w} \in K\left(\beta, n C, \frac{n C}{\rho}\right)$, that is, $w_{i} \in K_{i}\left(\beta_{i}, C, \frac{C}{\rho}\right)$, we have $\left\|w_{i}\right\|_{\infty} \leq \frac{C}{\rho}$, and from the definition of $K_{i}$, we know that $\min _{t \in J_{\rho}} w_{i}(t) \geq \rho\left\|w_{i}\right\|_{\infty}$. Thus we have

$$
\begin{aligned}
\beta\left(\mathbf{T}^{\boldsymbol{\Lambda}} \mathbf{w}\right) & =\sum_{i=1}^{n} \beta_{i}\left(T_{i}^{\lambda_{i}} \mathbf{w}\right)=\sum_{i=1}^{n} \min _{t \in J_{\rho}} T_{i}^{\lambda_{i}} \mathbf{w}(t) \\
& =\sum_{i=1}^{n} \min _{t \in J_{\rho}} \lambda_{i} \int_{0}^{1} G(t, s) h_{i}(s) f_{i}(\mathbf{w}(s)) d s \\
& \geq \sum_{i=1}^{n} \min _{t \in J_{\rho}} \lambda_{i}\left(\rho \int_{0}^{1} G(s, s) \eta_{i} f_{i}(\mathbf{w}(s)) d s\right) \\
& >\sum_{i=1}^{n} \frac{1}{6} \lambda_{i} \rho \eta_{i} \frac{C}{N_{i}} \\
& =n C .
\end{aligned}
$$

This implies that condition (i) of Lemma 4.4 holds.

For $w_{i} \in \bar{K}_{i, M}$, we have

$$
\begin{aligned}
\left\|\mathbf{T}^{\boldsymbol{\Lambda}} \mathbf{w}\right\| & =\sum_{i=1}^{n}\left\|T_{i}^{\lambda^{i}} \mathbf{w}\right\|_{\infty} \\
& =\sum_{i=1}^{n} \max _{t \in J} \lambda_{i} \int_{0}^{1} G(t, s) h_{i}(s) f_{i}(\mathbf{w}(s)) d s \\
& \leq \sum_{i=1}^{n} \max _{t \in J} \lambda_{i} \int_{0}^{1} G(s, s) h_{i}(s) f_{i}(\mathbf{w}(s)) d s \\
& <\sum_{i=1}^{n} \lambda_{i} \int_{0}^{1} G(s, s) h_{i}(s) \frac{M}{D_{i}} d s \\
& =n M .
\end{aligned}
$$

This implies that condition (ii) of Lemma 4.4 holds.

Finally, we assert that if $w_{i} \in K_{i}\left(\beta_{i}, C, L\right)$ and $\left\|T_{i}^{\lambda_{i}} \mathbf{w}\right\|_{\infty}>\frac{C}{\rho}$, then $\beta\left(\mathbf{T}^{\Lambda} \mathbf{w}\right)>n C$.

Suppose that $w_{i} \in K_{i}\left(\beta_{i}, C, L\right)$ and $\left\|T_{i}^{\lambda_{i}} \mathbf{w}\right\|_{\infty}>\frac{C}{\rho}$. Then

$$
\begin{aligned}
\beta\left(\mathbf{T}^{\Lambda} \mathbf{w}\right) & =\sum_{i=1}^{n} \beta_{i}\left(T_{i}^{\lambda_{i}} \mathbf{w}\right)=\sum_{i=1}^{n} \min _{t \in J_{\rho}} T_{i}^{\lambda_{i}} \mathbf{w}(t) \\
& =\sum_{i=1}^{n} \min _{t \in J_{\rho}} \lambda_{i} \int_{0}^{1} G(t, s) h_{i}(s) f_{i}(\mathbf{w}(s)) d s \\
& \geq \sum_{i=1}^{n} \rho\left\|T_{i}^{\lambda_{i}} \mathbf{w}\right\|_{\infty}>n C .
\end{aligned}
$$

This implies that condition (iii) of Lemma 4.4 holds. 
To sum up, the hypotheses of Lemma 4.4 hold. Therefore, an application of Lemma 4.4 implies that system (1.1) has at least three positive radial solutions $\mathbf{w}_{1}, \mathbf{w}_{2}, \mathbf{w}_{3}$ satisfying

$$
\left\|\mathbf{w}_{1}\right\|<n M, \quad n C<\beta\left(\mathbf{w}_{2}\right), \quad\left\|\mathbf{w}_{3}\right\|>n M, \quad \beta\left(\mathbf{w}_{3}\right)<n C .
$$

The proof is finished.

\subsection{An example}

Example 4.1 We consider the example $(n=2)$

$$
\left\{\begin{array}{l}
\Delta \mathbf{u}(\mathbf{x})+\boldsymbol{\Lambda} \mathbf{a}(|\mathbf{x}|) \mathbf{f}(\mathbf{u}(\mathbf{x}))=\mathbf{0}, \quad R_{1}<|\mathbf{x}|<R_{2}, \\
\left.\mathbf{u}\right|_{|\mathbf{x}|=R_{1}}=\left.\mathbf{u}\right|_{|\mathbf{x}|=R_{2}}=\mathbf{0}
\end{array}\right.
$$

By appropriate transformations, (4.8) can be written

$$
\begin{cases}w_{1}^{\prime \prime}(t)+\lambda_{1} h_{1}(t) f_{1}(\mathbf{w})=0, & 0<t<1, \\ w_{2}^{\prime \prime}(t)+\lambda_{2} h_{2}(t) f_{2}(\mathbf{w})=0, & 0<t<1, \\ w_{1}(1)=w_{1}(0)=w_{2}(1)=w_{2}(0)=0 .\end{cases}
$$

Let

$$
\begin{aligned}
h_{1}(t)= \begin{cases}\frac{1}{2}-t, & t \in\left[0, \frac{1}{2}\right), \\
t-\frac{1}{2}, & t \in\left[\frac{1}{2}, 1\right],\end{cases} & h_{2}(t)= \begin{cases}\frac{1}{4}-t^{2}, & t \in\left[0, \frac{1}{2}\right), \\
t^{2}-\frac{1}{4}, & t \in\left[\frac{1}{2}, 1\right],\end{cases} \\
f_{1}(\mathbf{w})=w_{1}^{2}, \quad f_{2}(\mathbf{w})=w_{2}^{3}, & \mathbf{w}=\left(w_{1}, w_{2}\right)^{T} .
\end{aligned}
$$

It is clear that $\xi=\frac{1}{2}, \sigma=\frac{1}{3}, c_{1}=c_{2}=1, k_{1}=k_{2}=1, l_{1}=l_{2}=2, \delta(t)=\min \left\{\frac{t}{\xi}, \frac{\xi-t}{\xi}\right\}=\min \{2 t, 1-$ $2 t\}, t \in[0, \xi], \alpha=\min \frac{\sigma}{2} \leq t \leq \sigma \delta(t)=\frac{1}{3}$. Let $r=\frac{1}{5}$. Then

$$
\begin{aligned}
& f_{1, r}=\min \left\{f_{1}(\mathbf{w}): \alpha r \leq w_{j} \leq r, j=1,2, \ldots, n\right\}=\frac{1}{225} \\
& f_{2, r}=\min \left\{f_{1}(\mathbf{w}): \alpha r \leq w_{j} \leq r, j=1,2, \ldots, n\right\}=\frac{1}{3375}
\end{aligned}
$$

From the above, let $\lambda_{1,0}=\frac{311,040}{13}, \lambda_{2,0}=\frac{209,952,000}{437}$. By Theorem 3.1, system (4.8) has at least two positive radial solutions for $\lambda_{i} \in\left[\lambda_{i, 0},+\infty\right), i=1,2$.

\section{Acknowledgements}

We wish to express our gratitude to Prof. Xuemei Zhang, School of Mathematics and Physics, North China Electric Power University, Beijing, PR China, for her kind help, careful reading, and for making useful comments on the earlier version of this paper. The authors are also grateful to the anonymous referees for their constructive comments and suggestions, which has greatly improved this paper.

\section{Funding}

This work is sponsored by the National Natural Science Foundation of China (11301178), the Beijing Natural Science Foundation of China (1163007), the key research and cultivation project of the improvement of scientific research level of BISTU (2018ZDPY18/521823903), and the teaching reform project of BISTU (2018JGYB32). 
Ethics approval and consent to participate

Not applicable.

\section{Competing interests}

The authors declare that there is no conflict of interest regarding the publication of this manuscript. The authors declare that they have no competing interests.

\section{Consent for publication}

Not applicable.

\section{Authors' contributions}

The authors contributed equally to this article. They have all read and approved the final manuscript.

\section{Publisher's Note}

Springer Nature remains neutral with regard to jurisdictional claims in published maps and institutional affiliations.

Received: 16 April 2019 Accepted: 20 August 2019 Published online: 02 September 2019

\section{References}

1. Hao, X., Zuo, M., Liu, L.: Multiple positive solutions for a system of impulsive integral boundary value problems with sign-changing nonlinearities. Appl. Math. Lett. 82, 24-31 (2018)

2. Chen, X., Zhang, X.: Existence of positive solutions for singular impulsive differential equations with integral boundary conditions on an infinite interval in Banach spaces. Electron. J. Qual. Theory Differ. Equ. 4529 (2011)

3. Jiao, L., Zhang, X.: A class of second-order nonlocal indefinite impulsive differential systems. Bound. Value Probl. 2018, $163(2018)$

4. Zhang, X.: Exact interval of parameter and two infinite families of positive solutions for a $n$th order impulsive singular equation. J. Comput. Appl. Math. 330, 896-908 (2018)

5. Boucherif, A.: Second-order boundary value problems with integral boundary conditions. Nonlinear Anal. 70, 364-371 (2009)

6. Hao, X., Liu, L., Wu, Y., Xu, N.: Multiple positive solutions for singular nth-order nonlocal boundary value problems in Banach spaces. Comput. Math. Appl. 61, 1880-1890 (2011)

7. Zhang, X., Ge, W.: Symmetric positive solutions of boundary value problems with integral boundary conditions. Appl. Math. Comput. 219, 3553-3564 (2012)

8. Liu, L., Hao, X., Wu, Y.: Positive solutions for singular second order differential equations with integral boundary conditions. Math. Comput. Model. 57, 836-847 (2013)

9. Feng, M.: Existence of symmetric positive solutions for a boundary value problem with integral boundary conditions. Appl. Math. Lett. 24, 1419-1427 (2011)

10. Zhang, X., Feng, M.: Existence of a positive solution for one-dimensional singular $p$-Laplacian problems and its parameter dependence. J. Math. Anal. Appl. 413, 566-582 (2014)

11. Wei, Y., Bai, Z., Sun, S.: On positive solutions for some second-order three-point boundary value problems with convection term. J. Inequal. Appl. 2019, 72 (2019)

12. Karakostas, G.L., Tsamatos, P.C.: Multiple positive solutions of some Fredholm integral equations arisen from nonlocal boundary-value problems. Electron. J. Differ. Equ. 2002, 30 (2002)

13. Zhang, X., Feng, M.: Double bifurcation diagrams and four positive solutions of nonlinear boundary value problems via time maps. Commun. Pure Appl. Anal. 17, 2149-2171 (2018)

14. Ji, D., Bai, Z., Ge, W.: The existence of countably many positive solutions for singular multipoint boundary value problems. Nonlinear Anal. 72, 955-964 (2010)

15. Feng, M., Li, P., Sun, S.: Symmetric positive solutions for fourth-order $n$-dimensional $m$-Laplace systems. Bound. Value Probl. 2018, Article ID 63 (2018)

16. Zhang, X., Ge, W.: Positive solutions for a class of boundary-value problems with integral boundary conditions Comput. Math. Appl. 58, 203-215 (2009)

17. Kawano, N., Kusano, T.: On positive entire solutions of a class of second order semilinear elliptic systems. Math. Z. 186 287-297 (1984)

18. Benrhouma, M.: Existence of solutions for a semilinear elliptic system. ESAIM Control Optim. Calc. Var. 19, 574-586 (2013)

19. Benrhouma, M.: Existence and uniqueness of solutions for a singular semilinear elliptic system. Nonlinear Anal. 107, 134-146 (2014)

20. Chen, K.: Multiplicity for strongly indefinite semilinear elliptic system. Nonlinear Anal. 72, 806-821 (2010)

21. Costa, D.G.: On a class of elliptic systems in $R^{N}$. Electron. J. Differ. Equ. 1994, 7 (1994)

22. Clément, P., Figuereido, D.G., Mitidieri, E.: Positive solutions of semilinear elliptic systems. Commun. Partial Differ. Equ. 17, 923-940 (1992)

23. Lee, Y.: Multiplicity of positive radial solutions for multiparameter semilinear elliptic systems on an Annulus. J. Differ. Equ. 174, 420-441 (2001)

24. Dunninger, D.R., Wang, H.: Multiplicity of positive radial solutions for an elliptic system on an annulus. Nonlinear Anal. 42,803-811(2000)

25. Chen, S., Lu, G.: Existence and nonexistence of positive radial solutions for a class of semilinear elliptic system. Nonlinear Anal. 38, 919-932 (1999)

26. Busca, J., Sirakov, B.: Symmetry results for semilinear elliptic systems in the whole space. J. Differ. Equ. 163, 41-56 (2000)

27. Zhong, J., Chen, Z.: Existence and uniqueness of positive solutions to a class of semilinear elliptic systems. Acta Math. Sci. 22B, 451-458 (2002) 
28. Lair, A.V., Wood, A.W.: Existence of entire large positive solutions of semilinear elliptic systems. J. Differ. Equ. 164 380-394 (2000)

29. Miao, Q., Yang, Z:: On the existence of multiples positive entire solutions for a quasilinear elliptic systems. Appl. Math. Comput. 198, 12-23 (2008)

30. Zhang, X., Liu, L.: The existence and nonexistence of entire positive solutions of semilinear elliptic systems with gradient term. J. Math. Anal. Appl. 371, 300-308 (2010)

31. Hai, D.D.: Existence and uniqueness of solutions for quasilinear elliptic systems. Proc. Am. Math. Soc. 133, 223-228 (2005)

32. Bozhkov, Y., Mitidieri, E.: Existence of multiple solutions for quasilinear systems via fibering method. J. Differ. Equ. 190, 239-267 (2003)

33. Ghergu, M.: Lane-Emden systems with negative exponents. J. Funct. Anal. 258, 3295-3318 (2010)

34. Hernándandez, J., Mancebo, F.J., Vega, J.M.: Positive solutions for singular semilinear elliptic systems. Adv. Differ. Equ. $13,9-10(2008)$

35. Ding, L., Xiao, S.W.: Solutions for singular elliptic systems involving Hardy-Sobolev critical nonlinearity. Differ. Equ. Appl. 2, 227-240 (2010)

36. Colin, F., Frigon, M.: Systems of singular Poisson equations in unbounded domains. Adv. Differ. Equ. 10, 9 (2005)

37. Serrin, J., Zou, H.: Nonexistence of positive solutions of Lane-Emden systems. Differ. Integral Equ. 9, 635-653 (1996)

38. Dalmasso, R.: Existence and uniqueness of positive solutions of semilinear elliptic systems. Nonlinear Anal. 39, 559-568 (2000)

39. Precup, R.: Existence, localization and multiplicity results for positive radial solutions of semilinear elliptic systems. J. Math. Anal. Appl. 352, 48-56 (2009)

40. Maniwa, M.: Uniqueness and existence of positive solutions for some semilinear elliptic systems. Nonlinear Anal. 59, 993-999 (2004)

41. Mavinga, N., Pardo, R.: A priori bounds and existence of positive solutions for semilinear elliptic systems. J. Math. Anal. Appl. 449, 1172-1188 (2017)

42. Feng, M.: New results of coupled system of $k$-Hessian equations. Appl. Math. Lett. 94, 196-203 (2019)

43. Yao, Q.: Existence and multiplicity of positive radial solutions for a semilinear elliptic equation with change of sign. Appl. Anal. 80, 65-77 (2001)

44. Lee, Y: An existence result of positive solutions for singular superlinear boundary value problems and its applications. J. Korean Math. Soc. 34, 247-255 (1997)

45. Guo, D., Lakshmikantham, V:: Multiple solutions of two-point boundary value problems of ordinary differential equations in Banach spaces. J. Math. Anal. Appl. 129, 211-222 (1988)

46. Cheng, X., Zhang, Z:: Positive solutions for a multi-parameter system of second-order ordinary differential equations. Sci. China Math. 54, 959-972 (2011)

47. Wang, H.: On the number of positive solutions of nonlinear system. J. Math. Anal. Appl. 281, 287-306 (2003)

48. Guo, D., Lakshmikantham, V:: Nonlinear Problems in Abstract Cones. Academic Press, New York (1988)

\section{Submit your manuscript to a SpringerOpen ${ }^{\circ}$ journal and benefit from:}

- Convenient online submission

- Rigorous peer review

- Open access: articles freely available online

- High visibility within the field

- Retaining the copyright to your article

Submit your next manuscript at $\boldsymbol{~ s p r i n g e r o p e n . c o m ~}$ 